\title{
PmtA Regulates Pyocyanin Expression and Biofilm Formation in Pseudomonas aeruginosa
}

\author{
Amy V. Thees ${ }^{\dagger}$, Kathryn M. Pietrosimone ${ }^{\dagger}$, Clare K. Melchiorre, Jeremiah N. Marden, \\ Joerg Graf, Michael A. Lynes* and Michele Maltz-Matyschsyk*
}

Department of Molecular and Cell Biology, University of Connecticut, Mansfield, CT, United States

The opportunistic pathogen Pseudomonas aeruginosa expresses a small molecular weight, cysteine-rich protein (PmtA), identified as a metallothionein (MT) protein family member. The MT family proteins have been well-characterized in eukaryotes as essential for zinc and copper homeostasis, protection against oxidative stress, and the ability to modify a variety of immune activities. Bacterial MTs share sequence homology, antioxidant chemistry, and heavy metal-binding capacity with eukaryotic MTs, however, the impact of bacterial MTs on virulence and infection have not been well-studied. In the present study, we investigated the role of PmtA in P. aeruginosa PAO1 using a PmtAdeficient strain $(\Delta p m t A)$. Here we demonstrated the virulence factor, pyocyanin, relies on the expression of PmtA. We showed that PmtA may be protective against oxidative stress, as an alternative antioxidant, glutathione, can rescue pyocyanin expression. Furthermore, the expression of phzM, which encodes a pyocyanin precursor enzyme, was decreased in the $\Delta p m t A$ mutant during early stationary phase. Upregulated pmtA expression was previously detected in confluent biofilms, which are essential for chronic infection, and we observed that the $\Delta p m t A$ mutant was disrupted for biofilm formation. As biofilms also modulate antibiotic susceptibility, we examined the $\Delta p m t A$ mutant susceptibility to antibiotics and found that the $\Delta p m t A$ mutant is more susceptible to cefepime and ciprofloxacin than the wild-type strain. Finally, we observed that the deletion of pmtA results in decreased virulence in a waxworm model. Taken together, our results support the conclusion that PmtA is necessary for the full virulence of $P$. aeruginosa and may represent a potential target for therapeutic intervention.

Keywords: metallothionein, pyocyanin, biofilm, antibiotic resistance, virulence

\section{INTRODUCTION}

Pseudomonas aeruginosa is a non-fermenting Gram-negative, opportunistic pathogen that ranks amongst the top five hospital-associated infections. Most commonly, these bacteria are found in patients that are immunocompromised, have undergone invasive surgery and/or have underlying conditions such as diabetes (Moradali et al., 2017; Wong et al., 2021). P. aeruginosa infections frequently arise from associations with medical devices such as ventilators, central lines, urinary 
catheters and/or surgical/transplantation (Moradali et al., 2017) and have been implicated as the causative agent of a wide variety of infections (e.g., pneumonia, sepsis, keratitis, skin, bone, joint, endocarditis, and meningitis) (Gellatly and Hancock, 2013; Magalhães et al., 2016; Wong et al., 2021). $P$. aeruginosa infections associated with chronic lung disease such as cystic fibrosis disease, ventilator-associated pneumonia (VAP), and individuals with chronic obstructive pulmonary disease (COPD) are persistent and are often linked with an increase in morbidity and mortality (Moradali et al., 2017; Malhotra et al., 2019). More recently, P. aeruginosa has been found as a common coinfection in patients hospitalized with COVID-19 (Qu et al., 2021). A major challenge in effectively treating these infections is multidrug-resistant (MDR) strains of $P$. aeruginosa. MDR strains have been associated with increased visits to clinical settings, very poor clinical outcomes, and are considered critical on the priority list by the World Health Organization (Tacconelli et al., 2013; Shrivastava and Ramasamy, 2018; Malhotra et al., 2019). Intrinsic antimicrobial resistance genes make $P$. aeruginosa highly resistant to antibiotics. These genes include efflux pump systems, beta-lactamase production, porin alterations, and target site modifications (Chuanchuen et al., 2002; Aedekerk et al., 2005; Reygaert, 2018). Although conventional antibiotics such as cefepime and ciprofloxacin are currently used to treat $P$. aeruginosa infections, resistance rates continue to rise in the four major groups of anti-pseudomonal agents: carbapenem, aminoglycosides, cephalosporins, and fluoroquinolones (Marvig et al., 2014; Pachori et al., 2019; Gajdács et al., 2021). Therefore, without reasonable availability of new therapeutic agents, drug resistance may worsen. Recently, targeting virulence factors has emerged as a new line of action in combating MDR $P$. aeruginosa strains and has revealed 12 classes of smallmolecule inhibitors and two antibodies that attack key virulence regulators (Shao et al., 2020).

To establish an infection, $P$. aeruginosa uses an array of virulence factors to counteract host defenses, cause direct damage to the host tissues and increase microbial competitiveness (Gellatly and Hancock, 2013). Some of these virulence factors involve phenazines, biofilms, exotoxins, endotoxins, proteases, siderophores, flagella, and secretion systems (Behzadi et al., 2021). Phenazines are secondary metabolites produced by Pseudomonas species that have been shown to increase microbial virulence (Das et al., 2016; Gonçalves and Vasconcelos, 2021). P. aeruginosa synthesizes a variety of these compounds, i.e., pyocyanin, phenazine-1carboxylic acid (PCA), 1-hydroxyphenazine (1-OH-PHZ) and phenazine1-carboxamine $(\mathrm{PCN})$. Construction of phenazines involves two homologous core loci biosynthetic pathways (phzA1B1C1D1E1F1G1 and phzA2B2C2D2E2F2G2) along with three genes ( $p h z M, p h z S$ and $p h z H$ ) that encode enzymes responsible for converting PCA to pyocyanin, PCN and 1-OH-PHZ. Pyocyanin is a well-characterized virulence factor produced by $95 \%$ of $P$. aeruginosa isolates (Gonçalves and Vasconcelos, 2021). Importantly, this oxidant-sensitive metabolite allows $P$. aeruginosa to manipulate the redox micro-environment during infection. Pyocyanin induces reactive oxygen species (ROS) production by host cells and blocks the host expression of catalase, an enzyme that neutralizes ROS through NADPH oxidation (Lau et al., 2004a,b). Sustained exposure to ROS results in host cell damage and a decrease in host defenses, which allows bacteria to establish a chronic infection (Gellatly and Hancock, 2013). In a murine infection model, pyocyanin-deficient mutants are more readily cleared from the lungs and less able to cause pneumonia than their wild-type counterparts (Lau et al., 2004b). Taken together, these results suggest that pyocyanin expression is essential for disease.

Another important virulence factor for $P$. aeruginosa is the ability to form biofilms. Biofilms are complex communities of microbes encapsulated in an extracellular polymeric substance (EPS) matrix that is comprised of proteins, lipids and extracellular DNA. Extracellular DNA (eDNA) has been identified as a major component of $P$. aeruginosa biofilm that is released by the lysing of a subpopulation of $P$. aeruginosa cells. In $P$. aeruginosa, eDNA release is mediated by quorum sensing (QS) signaling molecules $N$-acyl-L-homoserine lactone and Pseudomonas quinolone. Mediation of eDNA can also be affected by flagella and type IV pili mechanisms. In addition to initiating biofilm formation, eDNA can also bind to antibiotics, affording biofilm-associated cells even greater protection from these molecules. Pyocyanin is also regulated by QS and is essential for the release of eDNA, aiding in the formation of biofilms (Das et al., 2013).

Pseudomonas aeruginosa expresses a metallothionein (MT) known as PmtA ( $\underline{P}$. aeruginosa MT A) (Blindauer, 2008). Although little is known regarding the function of bacterial MTs, they do share some sequence homology, heavy metal binding characteristics, and cysteine motifs with their eukaryotic counterparts (Figure 1A and Table 1). Eukaryotic MTs are well studied in mammals for their immunomodulatory role. MTs can influence leukocyte trafficking and decrease proinflammatory mediators (Youn et al., 1995; Yin et al., 2005). Additionally, MT expression intensifies inflammatory bowel disease, alters the antibody response to antigen challenge, and anti-MT antibodies can ameliorate the severity of chronic inflammation (Lynes et al., 1993; Laukens et al., 2009; Devisscher et al., 2014).

A classic feature of the MT protein family is an abundance of cysteine residues and the presence of free thiol groups. These allow MTs to bind divalent heavy metals and contribute to their immunomodulatory functions (Lynes et al., 1993). MTs protects the host from ROS-induced damage during oxidative stress through oxidation of thiol-groups, which may allow macrophages to produce more ROS in the presence of MTs (Borghesi and Lynes, 1996). PmtA may have similar immunomodulatory properties and potentially influence Pseudomonas virulence due to its similarities in structure to mammalian MT. PmtA has also been shown to be upregulated in confluent biofilms compared to planktonic cells (Hentzer et al., 2005; Waite et al., 2006).

To date, the function of PmtA in $P$. aeruginosa infections in relation to virulence is unknown. In this study, we investigated the role of PmtA in $P$. aeruginosa strain PAO1, using a clean deletion mutant $(\Delta p m t A)$. We also investigated the role 
A

Alignment of Homo sapien MT-1 and P. aeruginosa PAO1 PmtA

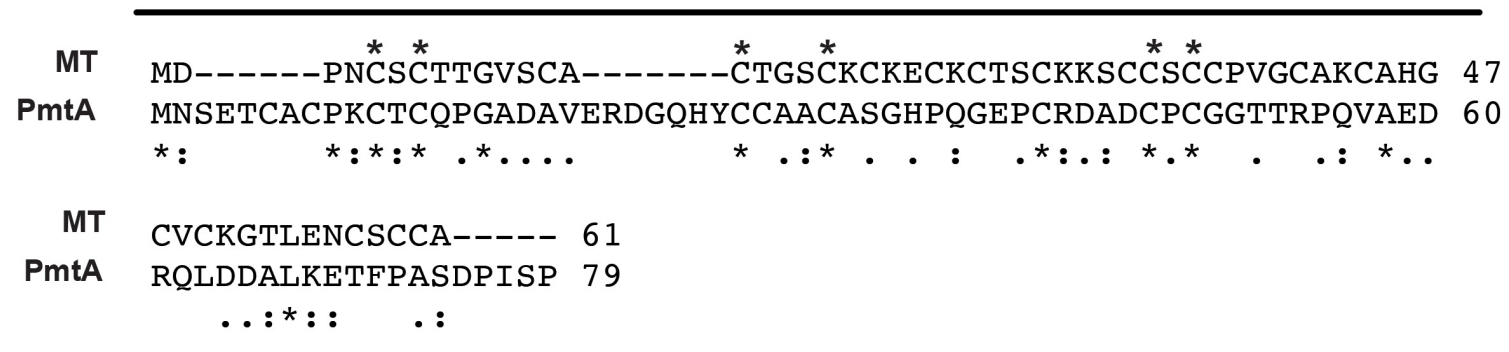

B
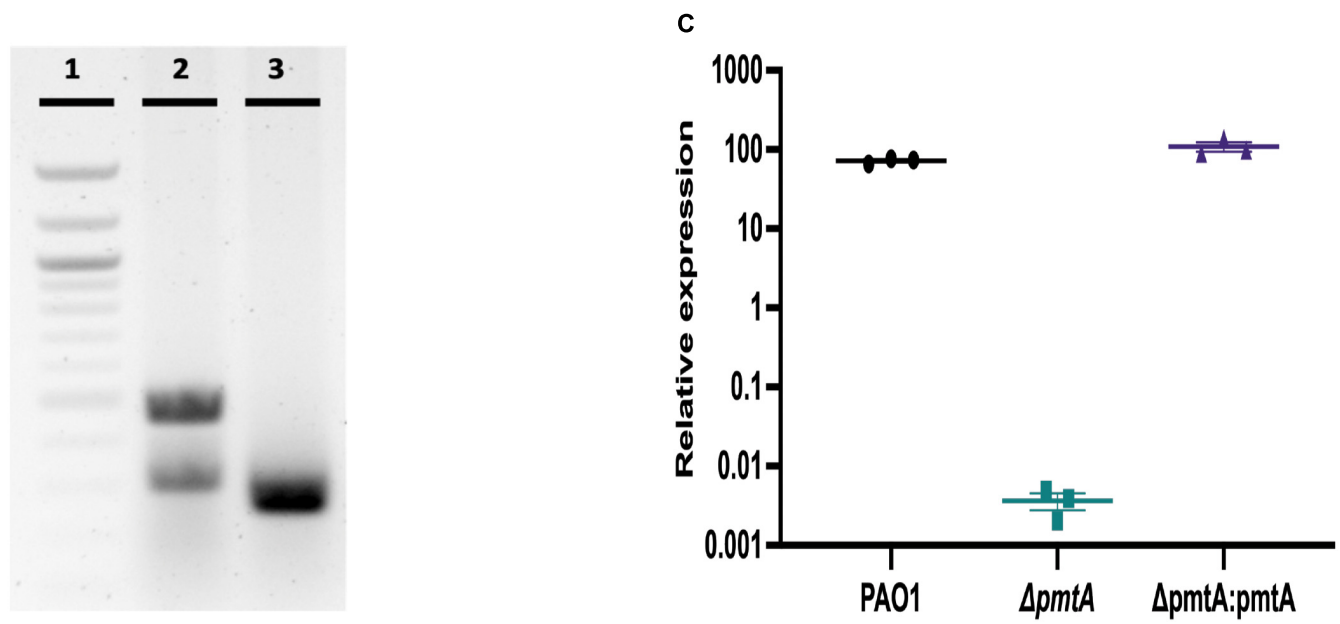

FIGURE 1 | Comparison of PAO1 PmtA and Homo sapiens Metallothionein I and generation valuation of the $\triangle$ pmtA mutant. (A) MT from P. aeruginosa PAO1 contains 79 amino acids, 10 of which are cysteine residues. A comparison of human MT-1 (Accession: AAH29475) and PAO1 PmtA (Accession: NP_250830) using the BLAST online software reveals there is little homology between the sequences $(e=6)$, but the alignment reveals many of the fully conserved regions ${ }^{*}$, below sequence) are cysteine residues. A (:) indicates conservation of amino acids with strongly similar properties, while (.) indicates conservation of amino acids with somewhat similar properties. Cysteine residues that are conserved between MT-1 and PmtA are indicated by $\left({ }^{*}\right)$ above the sequence. (B) Confirmation of clean deletion mutant was revealed by colony PCR. Lane 1, shows a 100 bp ladder; lane 2, shows wild-type PAO1 (485 bp), and lane 3, shows the $\triangle$ pmtA mutant (263 bp). (C) Overnight cultures of $P$. aeruginosa strains were grown in LB media for $24 \mathrm{~h}$ and total RNA was extracted from the cell pellets. Relative expression values for $p m t A$ were calculated using the cycle threshold value compared to a standard curve for pmtA. pmtA expression was decreased in the $\triangle p m t A$ mutant. This expression was restored in the complemented strain, $\Delta p m t A$ :pmtA. One-way ANOVA analysis was performed $\left({ }^{*} p<0.05 ;{ }^{* \star} p<0.01 ;{ }^{* \star *} p<0.001 ;{ }^{* \star \star *} p<0.0001\right)$. The data are presented as the average of three biological replicates ( \pm standard error of the mean) and are representative of three separate experiments.

TABLE 1 | PmtA comparison of Heavy metal binding characteristics and cysteine motifs.

\begin{tabular}{lccc}
\hline & PmtA (Pseudomonas species) & SmtA (Synechococcus species) & Mammalian MT \\
\hline \# of amino acids & $56-79$ & $55-56$ & $60-68$ \\
\# of cysteine residues & $8-10$ & $8-10$ & $18-23$ \\
\# of bound zinc & $3-4$ & $3-4$ & $7-8$ \\
\hline
\end{tabular}

PmtA plays in biofilm formation, pyocyanin production and antibiotic sensitivity.

\section{MATERIALS AND METHODS}

\section{Bacterial Strains, Plasmids, Growth Media, and Antibiotics}

The bacterial strains, plasmids, and primers used in the present study are listed in Supplementary Tables S1, S2.
P. aeruginosa strain PAO1 (Jacobs et al., 2003) was used as the wild-type strain, and all $P$. aeruginosa strains were grown at $37^{\circ} \mathrm{C}$ in Luria-Bertani (LB) or M9 salt liquid media or on agar-solidified plates. M9 salts $(5 \times)$ contained (per liter) $30 \mathrm{~g}$ of $\mathrm{Na}_{2} \mathrm{HPO}_{4}, 15 \mathrm{~g}$ of $\mathrm{KH}_{2} \mathrm{PO}_{4}, 5 \mathrm{~g}$ of $\mathrm{NH}_{4} \mathrm{Cl}$, and $2.5 \mathrm{~g}$ of $\mathrm{NaCl}$. M9-based medium $6.25 \mathrm{~mL}$ of $5 \times \mathrm{M} 9$ salts, $625 \mu \mathrm{L}$ of $20 \%$ glucose, $62 \mu \mathrm{L}$ of $1 \mathrm{M} \mathrm{MgSO}_{4}$, $3.25 \mu \mathrm{L}$ of $1 \mathrm{M} \mathrm{CaCl}_{2}$ and $24.3 \mathrm{~mL}$ of Nanopure water. The media were sterilized through $0.2 \mu \mathrm{m}$ sized filters into 50$\mathrm{mL}$ sterile falcon tubes and supplemented with $100 \mu \mathrm{g} / \mathrm{mL}$ 
gentamicin (GBiosciences St. Louis, MO, United States, RC176) as needed.

\section{Deletion and Complementation of PmtA}

An unmarked deletion mutant $(\triangle p m t A)$ was derived from PAO1 using a two-step allelic exchange as previously described (Hoang et al., 1998; Hmelo et al., 2015) and depicted in Supplementary Figure S1. Briefly, using the primer pairs 1_PA2140_del_F/R and 2_PA2140_del_F/R, approximately 500-bp fragments upstream and downstream of the target genes were amplified with 1520 bp overlapping end complimentary to BamHI-HF (New England BioLabs (NEB), Ipswich MA, R3136S) and EcoRIHF (NEB, R3101S)-digested pEX18Gm using Q5 High-Fidelity DNA Polymerase (NEB, M0491S). The mutant gene fragments were assembled using Gibson assembly (NEB, E5510S) with double-digested pEX18Gm and transformed into One Shot PIR1 (Invitrogen, Waltham, MA, United States, C101010) chemically competent Escherichia coli cells. Transformants were selected on LB plates supplemented with $15 \mu \mathrm{g} / \mathrm{mL}$ gentamicin and screened using colony PCR with the primer pair 2_PA2140_test_F/R. Clones bearing allelic exchange vectors, in which the mutant allele was inserted, were then assessed for correctness using Sanger sequencing. Plasmids were purified, transformed into donor E. coli strain SM10 $\lambda$-pir and conjugated into P. aeruginosa PAO1 recipient cells. Transformants were selected on VogelBonner minimal media containing $10 \mathrm{mM}$ citrate as a carbon source to select against E. coli. To select against merodiploids, LB agar containing $15 \%\left(\mathrm{wt} / \mathrm{vol}\right.$ ) sucrose and no $\mathrm{NaCl}_{2}$ was used for counterselection. Pseudomonas isolation agar containing $60 \mu \mathrm{g} / \mathrm{mL}$ gentamicin was used to select for isolates having undergone spontaneous excision of the plasmid. Isolates that no longer could grow in the presence of gentamicin were screened for deletion of the wild-type allele using the primer pair PA2140_Tn7_F/R by colony PCR (Figure 1B) and assessed for correctness by Sanger sequencing.

A complemented deletion mutant strain $(\triangle p m t A: p m t A)$ was generated with pUC18R6K-mini-Tn7T-Gm (Addgene, Watertown, MA, United States, 65022) as described by Choi and Schweizer (2006). The full-length of the target gene (including a potential promoter) was amplified from $P$. aeruginosa strain PAO1 using the primer pairs PPA2140_Tn7_F/PA2140_Tn7_R. Amplification fragments were assembled using Gibson assembly with PstI-HF (NEB, R3140S) and KpnI-HF (NEB, R3142S)digested pUC18R6K-mini-Tn7T-Gm and transformed into One Shot PIR1 chemically competent E. coli cells for directed recombination of the fragment into the plasmid. The $\Delta p m t A$ mutant was transformed with the suicide plasmid and selected for on $\mathrm{LB}$ medium containing $60 \mu \mathrm{g} / \mathrm{mL}$ of gentamicin. Isolates that grew in the presence of gentamicin were screened for the presence of the complementation construct using the primer pair pTn7GM_F/R by colony PCR (Supplementary Figure S4) and assessed for correctness by Sanger sequencing.

\section{Bacterial Growth}

Overnight cultures were diluted 1:100 in fresh LB or M9 salt media without antibiotics, diluted and aliquoted into a 96-well plate (Greiner Bio-One, Monroe, NC, United States, 655101) in triplicate and then monitored for growth by measuring the optical density at $600 \mathrm{~nm}\left(\mathrm{OD}_{600}\right)$ in a Spectramax microplate reader (Molecular Devices, Sunnyvale, CA, United States). As $P$. aeruginosa aggregates after the first $4 \mathrm{~h}$ of cultivation, the strains were diluted 1:10 in LB media and vortexed before the $\mathrm{OD}_{600}$ was determined. For $P$. aeruginosa growth curves in LB supplemented with oxidants and metals, overnight cultures were diluted 1:100 in fresh LB medium and grown for $2 \mathrm{~h}$ to exponential phase. Then, $600 \mu \mathrm{L}$ of each culture was added in triplicate to the wells of a 24-well sterile plate (Fisher Scientific, Waltham, MA, United States, Cat\#353047) together with $10 \mathrm{mM}$ hydrogen peroxide, $100 \mu \mathrm{M} \mathrm{CdCl}_{2}, 200 \mu \mathrm{M} \mathrm{ZnCl}_{2}$, or medium. The strains were cultured for 10-12 h with intermittent shaking at $37^{\circ} \mathrm{C}$, after which growth was calculated by dividing the average $\mathrm{OD}_{600}$ value of triplicate cultures grown with oxidant by that obtained for cultures grown without oxidant. For M9 salt medium, overnight cultures were subcultured to an $\mathrm{OD}_{600}$ of 0.08 , and $600 \mu \mathrm{L}$ was added to the wells of a 24 -well sterile plate (Falcon, Cat\#353047) in triplicate and mixed with $400 \mu \mathrm{L}$ of M9 salt medium alone or supplemented with oxidants or metals $(200 \mu \mathrm{g} / \mathrm{mL}$ transferrin, $150 \mu \mathrm{M}$ ethylenediaminediacetic acid (EDDA), $50 \mu \mathrm{M} \mathrm{FeCl}_{2}, 10 \mathrm{mM}$ hydrogen peroxide, $200 \mu \mathrm{M}$ $\mathrm{CdCl}_{2}, 100 \mu \mathrm{M} \mathrm{ZnCl}$ ). The strains were cultured for $18 \mathrm{~h}$ with intermittent shaking at $37^{\circ} \mathrm{C}$. To measure pyocyanin production, single colonies of the $P$. aeruginosa strains were grown overnight in $5 \mathrm{~mL}$ of LB medium with the appropriate antibiotics at $37^{\circ} \mathrm{C}$ with vigorous shaking. These cultures were then diluted at 1:100 in fresh LB media without antibiotics at a dilution of $1: 100$ and grown at $37^{\circ} \mathrm{C}$ with vigorous shaking. Pyocyanin concentrations were determined as previously described (Frank and Demoss, 1959) with the exception that $1 \mathrm{~mL}$ of each culture was centrifuged for $1 \mathrm{~min}$ at $20,500 \times g$. The supernatants were transferred to new microcentrifuge tubes, vortexed vigorously and then transferred to a 96-well plate in triplicate to measure the absorbance at $691 \mathrm{~nm}$, with measurements taken at selected time points over $30 \mathrm{~h}$ of cultivation. The $\mathrm{OD}_{600}$ values of the cultures were also monitored, and data was plotted as $\mathrm{OD}_{600}$ vs. $\mathrm{Abs}_{691}$.

\section{Pyocyanin Extraction}

Pseudomonas aeruginosa strains were grown for $30 \mathrm{~h}$ in triplicate, and $1 \mathrm{~mL}$ of cell-free supernatants were obtained. Pyocyanin was extracted as previously described with minor modifications (Frank and Demoss, 1959; Essar et al., 1990; Zaborin et al., 2012). In brief, $500 \mu \mathrm{L}$ of chloroform was added to $1 \mathrm{~mL}$ of supernatant from each strain. The mixture was then vigorously vortexed for $2 \mathrm{~min}$ as the chloroform turned blue green, after which the tubes were centrifuged for $10 \mathrm{~min}$ at $3000 \times g$ and $4^{\circ} \mathrm{C}$. Hydrochloric acid $(150 \mu \mathrm{L}, 0.2 \mathrm{M})$ was added to the partially extracted blue pyocyanin and vortexed vigorously for $2 \mathrm{~min}$. The top blue layer was acidified to a pink color and samples were centrifuged for $2 \mathrm{~min}$ at $3000 \times \mathrm{g}$ and $4^{\circ} \mathrm{C}$. The absorbance of completely extracted pyocyanin at the pink stage was measured at $520 \mathrm{~nm}$. Measurements were normalized to initial cell density $\mathrm{OD}_{600}$.

\section{RT-qPCR}

Pseudomonas aeruginosa strains were grown overnight in LB medium to stationary phase and a subculture was used to collect 
total RNA extracted from the cell pellets using QIAGEN RNeasy kit (74104) according to the manufacturer's recommendations at 4,10 , and $24 \mathrm{~h}$. RNA was converted to cDNA using the TaqMan Reverse Transcription kit (Invitrogen, N8080234). Gene-specific primers to $p m t A$ and the housekeeping ribosomal gene (RNA polymerase, subunit alpha) were designed and are listed in Supplementary Table S2. qPCR was performed on an Applied Biosystems 7300 instrument using iTaq Universal SYBR green (Bio-Rad, Hercules, CA, United States, 1725121). All qPCR experiments were performed with at least three biological replicates, each tested in triplicate for each time point. The absolute gene expression for $p m t A$ was calculated using the cycle threshold value compared to a standard curve of $p m t A$ using pGEX-6p-pmtA. The qPCR assay efficiency was verified using a five-fold dilution standard curve in triplicate. The $R^{2}$ of the standard curve was 0.99 and efficiency was 100\%. qPCR was performed on an Applied Biosystems 7300 instrument using iTaq Universal SYBR green (Bio-Rad, 1725121). All qPCR experiments were performed with at least three biological replicates, each tested in quadruplicate for each timepoint. The relative gene expression for $p h z H$ and $p h z M$ was calculated using the cycle threshold value compared to the ribosomal RNA gene as the internal reference standard. Fold changes were expressed as $2^{-\Delta \Delta \mathrm{Ct}}$ values. No template and wild-type PAO1 were used negative and positive control, respectively.

\section{Biofilm Formation Assay}

The wild-type, $\Delta p m t A$, and $\Delta p m t A: p m t A$ PAO1 strains were inoculated into $5 \mathrm{~mL}$ of $\mathrm{LB}$ and vigorously shaken at an angle in 14-mL Falcon tubes overnight. The medium was removed the next day, and the splash zone was stained for $15 \mathrm{~min}$ with $0.1 \%$ crystal violet (Fisher Science, Waltham, MA, United States, S25275A). Subsequently, the tubes were gently rinsed with water $(2 \times)$ and inspected for the presence of a biofilm. Biofilm formation was also quantified using a previously described microtiter plate assay with minor adaptations (O'Toole, 2010). In brief, overnight cultures were diluted at 1:10 in fresh LB medium and grown for $24 \mathrm{~h}$ at $37^{\circ} \mathrm{C}$ without shaking. Growth yields were determined by measuring the absorbance at $595 \mathrm{~nm}$. The medium was removed, and the biofilms were fixed to the sides of the microtiter wells with $90 \%$ methanol before being stained with $0.1 \%$ crystal violet (Fisher Science, Waltham, MA, United States, S25275A). The crystal violet was solubilized in ethanol:acetone $(4: 1, \mathrm{v} / \mathrm{v})$ and measured at $595 \mathrm{~nm}$ using an ethanol solution as blank. P. aeruginosa PAO1 was used as a positive control, with three biological replicates and eight technical replicates performed for each strain. The relative biofilm formation (RBF) for each well was determined by dividing the crystal violet absorbance reading by the growth yield absorbance reading.

\section{Acyl Homoserine Lactone-Dependent Quorum Sensing Assay}

The reporter strain E. coli MG4 pKDT17 (Addgene, Watertown, MA, United States, 27503) was used in crossfeeding assays with the wild-type, $\Delta p m t A$ or $\Delta p m t A: p m t A$ PAO1 strains and E. coli MC1061 (negative control) to assess acyl homoserine lactone (AHL) production as previously described (Pearson et al., 1995; Van Laar et al., 2018). In brief, wild-type, $\Delta p m t A$, and $\Delta p m t A: p m t A$ PAO1 strains and E. coli MG4 were grown overnight. Subsequently, each PAO1 strain was streaked onto a MacConkey agar plate $0.75 \mathrm{~cm}$ from a streak of E. coli MG4 and incubated overnight at $37^{\circ} \mathrm{C}$. The next day, the plates were examined for color change, where pink or purple indicated the presence of AHLs and white indicated their absence.

\section{Minimum Inhibitory Concentration Determination Using E-Test}

The susceptibility of $P$. aeruginosa strains to ciprofloxacin, cefepime and tetracycline, was assessed using Liofilchem ${ }^{\circledR}$ MTS $^{\mathrm{TM}}$ E-tests (Fisher Scientific, Waltham, MA, United States, 22-777-785, 22-777-917, 22-777-902) following the manufacturer's protocol. In short, fresh $P$. aeruginosa colonies were resuspended in $1 \mathrm{~mL}$ of $\mathrm{LB}$, and a cotton swab was then used to spread the inoculated LB over LB agar plates. The plates were allowed to dry, and then the E-test strip was then placed onto the inculcated agar, with each strain assayed in triplicate. The plates were incubated overnight at $37^{\circ} \mathrm{C}$, and the minimum inhibitory concentration (MIC) was read the next day.

\section{In vivo Galleria mellonella Larvae Model of Infection}

Healthy Galleria mellonella larvae (waxworms) were purchased from Vanderhorst Wholesale, Inc. (St Marys, OH, United States) and briefly stored at $4^{\circ} \mathrm{C}$ until use. Injections were performed as described in Seed and Dennis (2008) with a few adaptations. Cultures of $P$. aeruginosa strains were grown overnight, and the ability of $10^{3} \mathrm{CFU} / \mathrm{mL}$ of each $P$. aeruginosa to cause melanization was assessed. Worms were randomly separated into groups and injected into the hindmost left proleg with $5 \mu \mathrm{L}$ of sterile $10 \mathrm{mM} \mathrm{MgSO}_{4}$ or $5 \mu \mathrm{L}$ of $10^{3} \mathrm{CFU} / \mathrm{mL}$ of the wild-type $\Delta p m t A$ or $\Delta p m t A: p m t A$ PAO1 strains using a $10-\mu \mathrm{L}$ Hamilton syringe (Hamilton Company, Reno, NV, United States) with a beveled needle. The inoculum $\left(10^{3} \mathrm{CFU} / \mathrm{mL}\right)$ was verified by plating, and the injected worms were incubated at $37^{\circ} \mathrm{C} 48 \mathrm{~h}$, with survival assessed at 20,24, 28, and $48 \mathrm{~h}$ post injection (Tsai et al., 2016).

\section{RESULTS}

\section{The pmtA Clean Deletion Mutant Has no Detectable Metallothionein Production}

Despite being highly conserved, little is known regarding the physiological function and expression of $p m t A$ during the life cycle of $P$. aeruginosa. We constructed a clean deletion mutation of $\triangle p m t A$ in wild-type $P$. aeruginosa PAO1 using a two-step allelic exchange method as well as a complemented strain $(\Delta p m t A: p m t A)$ by cloning $p m t A$ in a Tn7 and conjugating it into $\triangle p m t A$. We verified the genetic change in the $\Delta p m t A$ strain using colony PCR and Sanger sequencing (Figure 1B, Supplementary Figure S2 and data not shown). The colony PCR shows a band shift when comparing the products from the 
PAO1 (485 bp) and $\triangle p m t A$ (263 bp) strains, indicating success in generation of a clean mutation. RT-qPCR was also used to assess $p m t A$ transcription levels in the wild-type, $\Delta p m t A$ and $\Delta p m t A: p m t A$ PAO1 strains. The wild-type and $\Delta p m t A: p m t A$ strains had approximately equal amounts of transcript, while little to no $p m t A$ transcript was present in $\Delta p m t A$ mutant cultures at 4,10 , and $24 \mathrm{~h}$ (Figure 1C). These results also suggest that $p m t A$ is expressed throughout stationary phases in PAO1.

\section{The $\Delta p m t A$, and $\Delta p m t A: p m t A$ PAO1 Strains Have Similar Growth Kinetics}

The growth kinetics of the wild-type, $\Delta p m t A$, and $\Delta p m t A: p m t A$ PAO1 strains were examined in both LB and M9 salt media. While no significant differences in the growth rates were detected between the strains (Supplementary Figures S3A-C), the wildtype and $\triangle p m t A: p m t A$ strains turned the media green after $10 \mathrm{~h}$ of growth, while the $\triangle p m t A$ culture media remained yellow (Figure 2A). This color difference persisted to $30 \mathrm{~h}$ of growth and is likely due to a difference in the production of pyocyanin, which is known to be secreted by PAO1 and imparts a distinct bluish-green color to media (Hassan and Fridovich, 1980).

\section{The $\Delta p m t A$ Mutant Produces Less Pyocyanin Than Wild-Type PAO1}

We investigated the ability of the $\Delta p m t A$ mutant to produce pyocyanin by determining the absorbance values of cell-free culture supernatants at $691 \mathrm{~nm}$ in a time course assay (Rada and Leto, 2013). Pyocyanin levels were decreased in the culture supernatants of the $\Delta p m t A$ strain compared to the wild-type and $\Delta p m t A: p m t A$ strains starting at $10 \mathrm{~h}$ and remained lower than those of the wild-type strain throughout the assay (Figure 2B). The highest levels of pyocyanin expression by PAO1 are observed during stationary phase growth (Rada and Leto, 2013). We compared cell growth to pyocyanin levels $(691 / 600 \mathrm{~nm}$ ratio) for the various PAO1 strains, and the normalized pyocyanin levels were consistently higher in the wild-type and $\triangle p m t A: p m t A$ than in the $\triangle p m t A$ mutant (Figure 2C). QS molecules, phenazineproducing genes $(p h z A-G)$, and the gene $p h z M$ are involved in pyocyanin production at early stationary phase. Wild-type PAO1 starts to produce detectable pyocyanin at $10 \mathrm{~h}$ (Das et al., 2016; Figures $\mathbf{2 B}, \mathbf{C})$. These results indicate that $p m t A$ is necessary for optimum pyocyanin levels in $P$. aeruginosa and that the restoration of $p m t A$ expression is sufficient for re-establishing pyocyanin to normal levels.

Pyocyanin appears as different colors in media depending on the $\mathrm{pH}$ and oxidation status of the culture (Rada and Leto, 2013). Highly oxygenated cultures are green due to the accumulation of oxidized pyocyanin, whereas those that are not well oxygenated appear yellow due to reduced pyocyanin (Rada and Leto, 2013). To ensure that the 691/600 nm ratio of wild-type PAO1 was not higher than that of the $\Delta p m t A$ mutant due to a difference in the $\mathrm{pH}$ or oxidation status, pyocyanin was extracted with chloroform $/ \mathrm{HCl}$ after $24 \mathrm{~h}$ of growth, and the absorption readings of the supernatants were analyzed as previously described (Rada and Leto, 2013). Wildtype PAO1 extracts were significantly higher than the $\Delta p m t A$ mutant extracts, while extracts were restored to wild-type levels in the complemented mutant $\Delta p m t A$ :pmtA (Figure 2D). These results indicate that wild-type PAO1 produces more pyocyanin than the $\Delta p m t A$ mutant and the difference in the $691 \mathrm{~nm}$ readings is not due to a difference in the oxidation state of pyocyanin or the $\mathrm{pH}$ of the culture.

\section{Exogenous Addition of the Antioxidant Glutathione Restores Pyocyanin Production to Wild-Type Levels in the $\Delta$ pmtA Mutant}

Because the $\triangle p m t A$ mutant produces less pyocyanin, we hypothesize that this strain also has a reduction in antioxidant capacity. To test this hypothesis, the alternative antioxidant glutathione (GSH) was added to the growth media, and changes in pyocyanin production were monitored. The absorbance values at 691 and $600 \mathrm{~nm}$ were measured at $0,2,4,6,8,10,12$, 24 , and $30 \mathrm{~h}$ of growth and the $691 / 600 \mathrm{~nm}$ ratios were calculated (Figure 3A). The addition of biological levels of GSH restored pyocyanin to wild-type levels, indicating that PmtA alters the redox environment and influences pyocyanin production (Figure 3B; Meister and Anderson, 1983).

\section{PmtA Plays an Essential Role in Phenazine Production}

The phzA1 and phzA2 gene clusters encode enzymes required for phenazine synthesis to produce phenazine-1-carboxylic acid. $\mathrm{PhzH}$ converts phenazine-1-carboxylic acid to phenazine-1carboxamide, while PhzM converts phenazine-1-carboxylic acid to 5-methylphenazine-1-carboxylic acid betaine, which is later converted to pyocyanin by PhzS (Mavrodi et al., 2001). To elucidate the mechanism by which PmtA affects pyocyanin biosynthesis, we assessed the expression of $p h z H$ and $p h z M$ using real-time PCR (Figure 4). The expression of phzM but not $p h z H$ was decreased in the $\Delta p m t A$ mutant (Figure 4A), and $p h z M$ expression was restored in the complemented mutant $\Delta p m t A: p m t A$ at $4 \mathrm{~h}$. However, no differences were observed between the wild-type, $\Delta p m t A$ and $\triangle p m t A$ :pmtA strains at the later time points (10 and $24 \mathrm{~h}$ ). These results suggest that PmtA biosynthesis is required for the early expression of phzM but not $p h z H$ via unknown mechanisms and that the expression levels of this gene contribute to the overall production of the pyocyanin precursor 5-methylphenazine-1-carboxylic acid betaine in $P$. aeruginosa.

\section{PmtA Is Essential for Biofilm Formation}

As upregulated PmtA expression has been observed in confluent $P$. aeruginosa biofilms (Hentzer et al., 2005; Waite et al., 2006), we investigated the role of PmtA in biofilm formation. First, we assessed biofilm formation by the wild-type, $\Delta p m t A$, and $\Delta p m t A: p m t A$ PAO1 strains by examining the splash zones of overnight cultures stained with crystal violet. As shown in Figure 5A, distinct crystal violet-stained biofilms were formed by the wild-type and $\Delta p m t A: p m t A$ strains but not the $\Delta p m t A$ mutant. Additionally, a crystal violet microtiter plate biofilm assay was used to quantitate the biofilm formation defect 

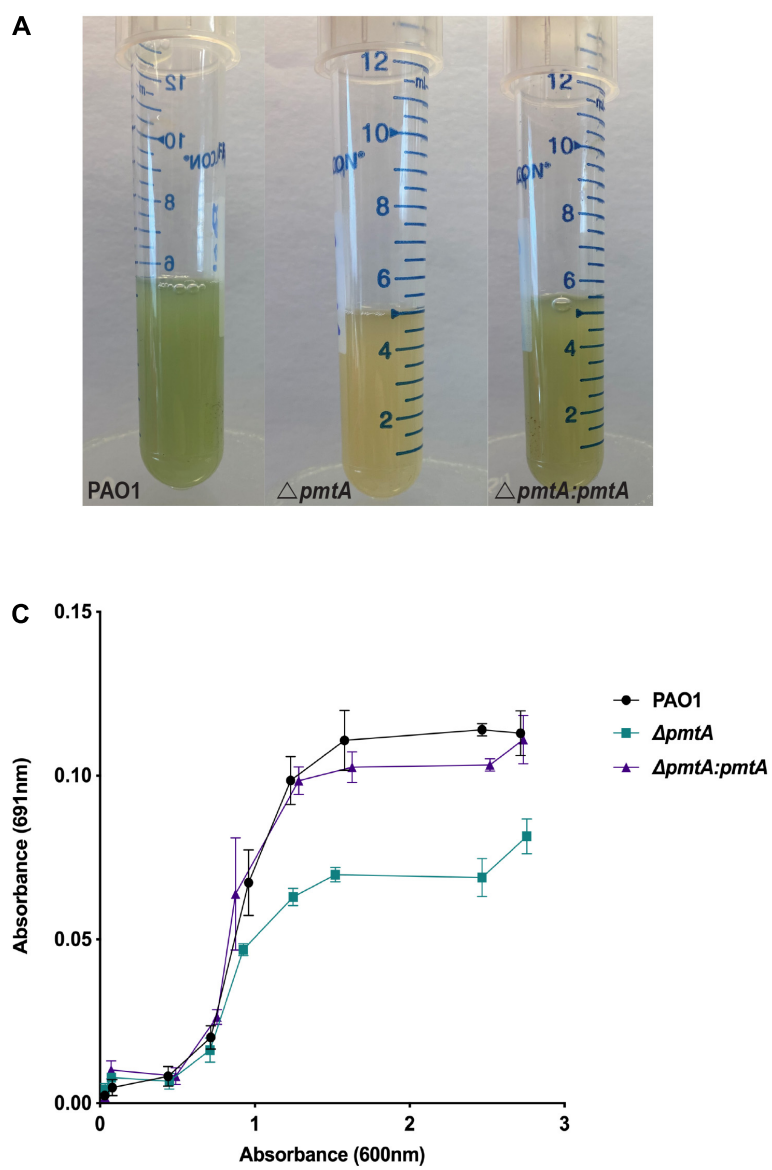

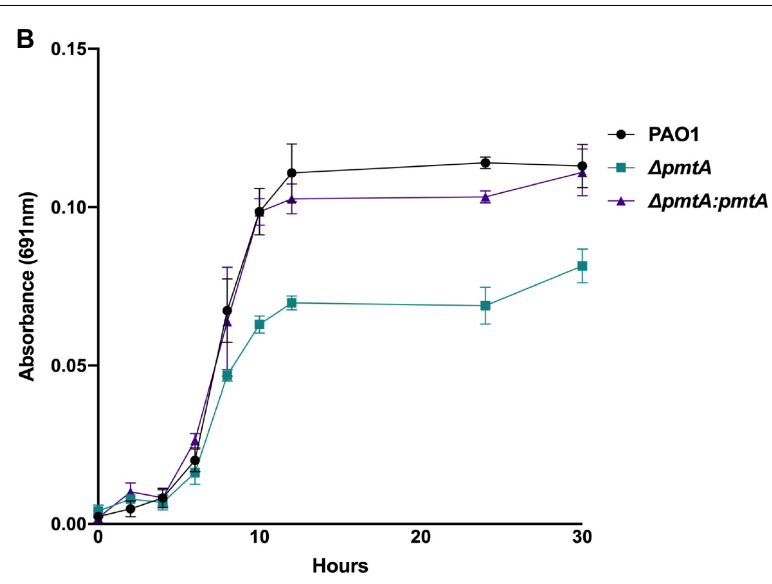

D

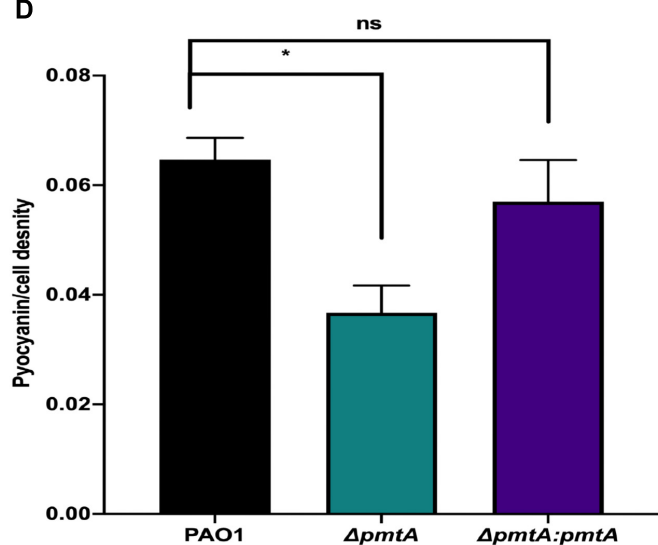

FIGURE $2 \mid P$. aeruginosa PAO1 strain $\Delta p m t A$ produces less pyocyanin than wild-type PAO1. (A) $P$. aeruginosa strains were each grown overnight in 5 mL of LB medium at $37^{\circ} \mathrm{C}$ for $24 \mathrm{~h}$ with vigorous shaking. The cultures were observed for color change and the $\Delta$ pmt $A$ mutant had a yellow appearance compared to wild-type PAO1. (B) Pyocyanin levels were measured at 0, 2, 4, 6, 8, 10, 12, 24, and $30 \mathrm{~h}$ using $1 \mathrm{~mL}$ of the culture and centrifuged for $1 \mathrm{~min}$ at 20,500 $\times \mathrm{g}$. The supernatant was then placed in a 96 well plate in triplicate and the absorbance at $691 \mathrm{~nm}$ was measured. (C) The data was plotted as $\mathrm{OD}_{600}$ vs. OD 691 .

(D) P. aeruginosa strains were grown for $24 \mathrm{~h}$, and cell-free supernatants were obtained. Pyocyanin was extracted as previously described, with minor modifications (Essar et al., 1990; Zaborin et al., 2012). Chloroform was added to the supernatant from each strain, forming a blue bottom layer. Subsequently, hydrochloric acid $(1.5 \mathrm{~mL}, 0.2 \mathrm{M})$ was added to the layer altering the $\mathrm{pH}$ to 2 and pink in color. The absorbance of completely extracted pyocyanin was measured at $\mathrm{OD}_{520}$ and the pyocyanin/cell density was calculated by dividing the pyocyanin at $\mathrm{OD}_{520}$ by growth measured at OD 600 . One-way ANOVA analysis was performed ( ${ }^{*} p<0.05$; ${ }^{* \star} p<0.01 ;{ }^{* \star} p<0.001 ;{ }^{* \star \star} p<0.0001$. The data are presented as the average of three biological replicates ( \pm standard error of the mean) and are representative of three separate experiments.

presented by the $\Delta p m t A$ strain. Interestingly, the $\Delta p m t A$ mutant showed a $28 \%$ decrease in biofilm formation compared to the wild-type and $\triangle p m t A: p m t A$ PAO1 strains (Figure 5B). These results indicate that the lack of PmtA contributed to decreased biofilm production by the $\triangle p m t A$ mutant and that the restoration of PmtA production resulted in the reestablishment of biofilm formation. Incidentally, the addition of the antioxidant GSH restored biofilm formation in the $\Delta p m t A$ mutant, confirming that redox plays a role in $P$. aeruginosa biofilm formation (Figure 5C).

\section{PmtA Is Not Essential for Managing Oxidative or Metal Stress}

Low-molecular-weight thiols are essential for detoxifying oxidative stressors and ameliorating heavy metal stress (Van
Laar et al., 2018; Ulrich and Jakob, 2019). For example, the lowmolecular-weight thiol OspR can act as an oxidative stress sensor in $P$. aeruginosa. MTs can actively scavenge reactive oxygen and nitrogen species due to their high abundance of cysteine residues (Ruttkay-Nedecky et al., 2013). In a previous study, pmtA expression was shown to be upregulated upon $10 \mathrm{mM}$ $\mathrm{H}_{2} \mathrm{O}_{2}$ stimulation for 15 min compared to untreated controls (Goldová et al., 2011). Therefore, we assessed whether the lack of PmtA contributes to increased sensitivity to various oxidants and heavy metals. First, we characterized the growth of the $\Delta p m t A$ mutant compared to the wild-type and $\Delta p m t A: p m t A$ PAO1 strains in the presence of $10 \mathrm{mM} \mathrm{H}_{2} \mathrm{O}_{2}$. All three strains grew to similar levels in both LB and M9 salt media supplemented with $10 \mathrm{mM} \mathrm{H}_{2} \mathrm{O}_{2}$ (Supplementary Figure S4). These results indicate that PmtA alone does not detoxify peroxides and that other factors may contribute to this process. 


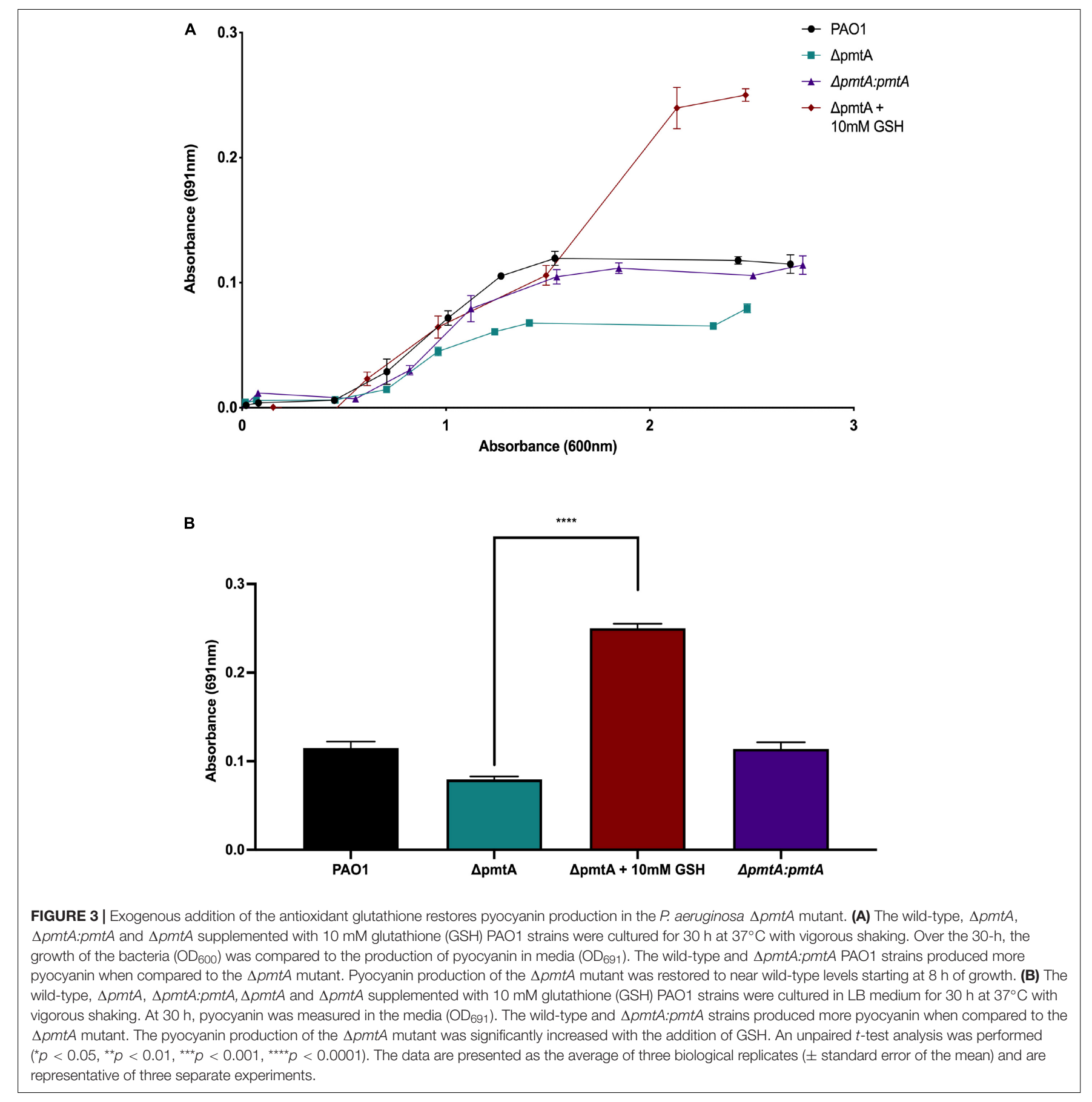

Zinc functions as a cofactor for many essential enzymes in all organisms. However, as excess zinc can be toxic, bacteria maintain intracellular zinc concentrations at low levels (Blindauer, 2015). Cadmium is toxic to most organisms and generates oxidative stress (Maret and Moulis, 2013). MTs play a role in zinc and copper homeostasis as well as cadmium detoxification (Blindauer, 2011). Cyanobacterial MTs are encoded within defined operons that include a zinc-dependent repressor $(\mathrm{SmtB})$ and an operator promoter region in the intergenic region (Morby et al., 1993; Blindauer,
2008, 2011). However, Pseudomonas MT-harboring operons appear to lack such features and encode proteins of unknown functions (Figure 1A and Table 1). In the present study, we investigated the role of PmtA in heavy metal resistance by culturing the wild-type, $\triangle p m t A$, and $\triangle p m t A: p m t A$ PAO1 strains in LB and in M9 salt media supplemented with zinc or cadmium and assessing their viability over time (Supplementary Figure S5). All strains grew to similar levels in both media types, with no differences observed between the strains exposed to inhibitory concentrations of $\mathrm{ZnCl}_{2}$ 


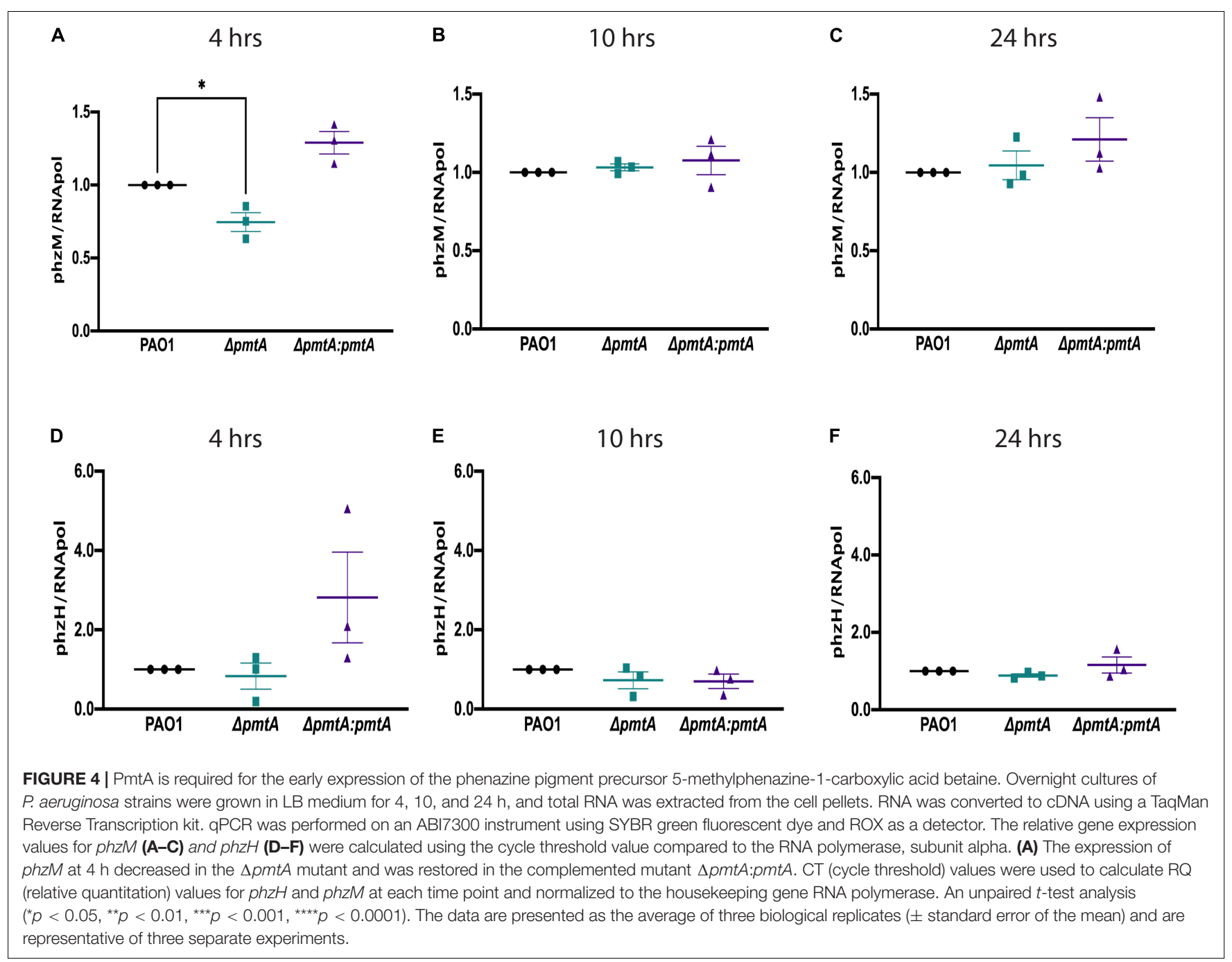

$(200 \mu \mathrm{M})$ (Supplementary Figures S5A,B) or $\mathrm{CdCl}_{2}(100 \mu \mathrm{M})$ (Supplementary Figures S5C,D). In addition, no differences were observed between the strains when cultured for $6 \mathrm{~h}$ before exposure (data not shown). The similar growth pattern of the strains under zinc or cadmium excess suggests that PmtA is not crucial for metal resistance.

As iron uptake is also associated with pyocyanin production, we examined the ability of the wild-type, $\Delta p m t A$, and $\Delta p m t A: p m t A$ strains to grow iron chelator EDDA. All strains had a reduced growth rate when compared to growth in M9 salt media indicating the iron limiting conditions. $\mathrm{FeCl}_{2}$ and transferrin restored the growth of all the stains showing that iron utilization is not affected by the loss of PmtA (Supplementary Figure S6).

\section{PmtA Does Not Inhibit Acyl Homoserine Lactone (AHL)-Mediated Quorum Sensing}

Quorum sensing has been shown to mediate many processes in $P$. aeruginosa, including pyocyanin production and biofilm formation. Therefore, we assessed whether AHL-dependent QS is altered in the $\triangle p m t A$ mutant using the reporter strain $E$. coli MG4 (pKDT17). In this strain, LasR transcribes a lasB-lacZ gene fusion in the presence of AHLs, and the resulting $\beta$-galactosidase fusion protein generates a color change in colonies from white to pink/purple when streaked on MacConkey agar. The wildtype, $\Delta p m t A$, and $\Delta p m t A: p m t A$ PAOl strains all induced a color change when streaked on MacConkey agar, indicating that AHL was produced and that PmtA does not mediate AHL-dependent QS (Figures 6A-C). As a negative control, strain E. coli MC1061 was also assessed, no color change was observed (Figure 6D).

\section{PmtA Promotes Antibiotic Resistance in PA01}

Weakened resistance to antibiotics is a classic biofilm defect characteristic. Therefore, we examined the sensitivity of the $\Delta p m t A$ mutant to tetracycline, to which $P$. aeruginosa is intrinsically resistant, and two of the conventional antibiotics used to treat CF patients infected with $P$. aeruginosa, cefepime and ciprofloxacin. Using test strips, we quantitatively assessed the MIC of the antibiotics on the wild-type, $\Delta p m t A$, and 


\section{A}

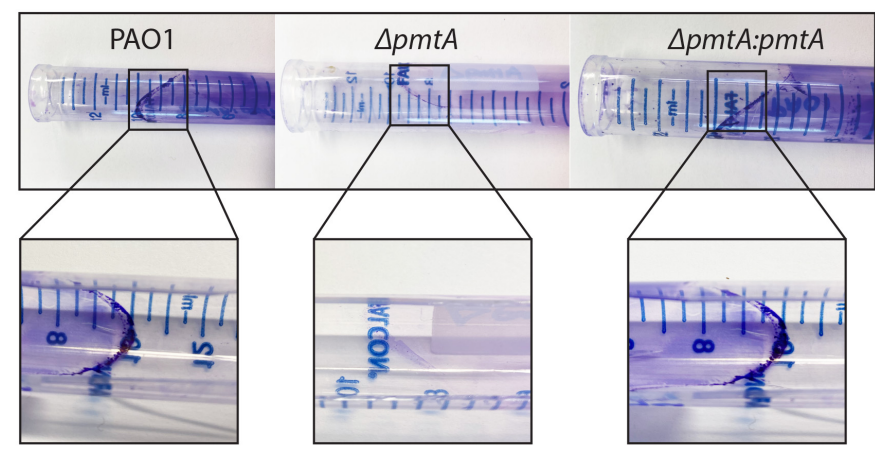

B

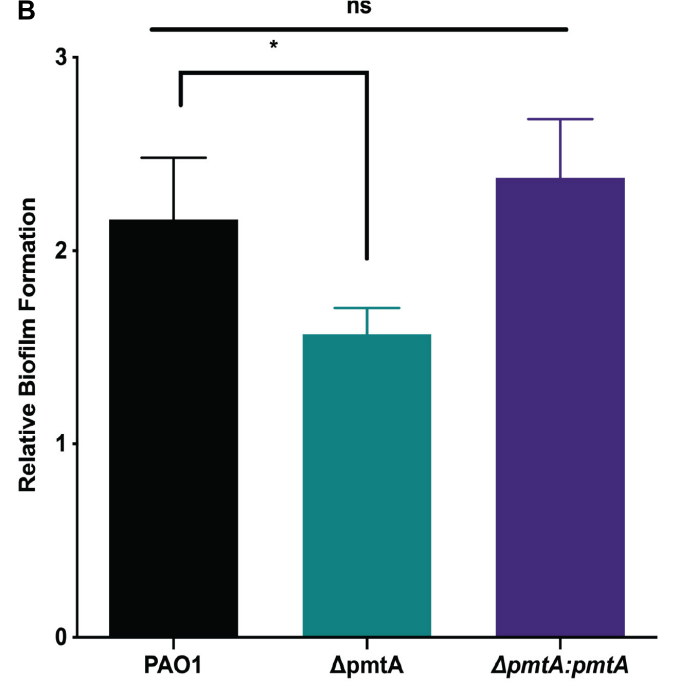

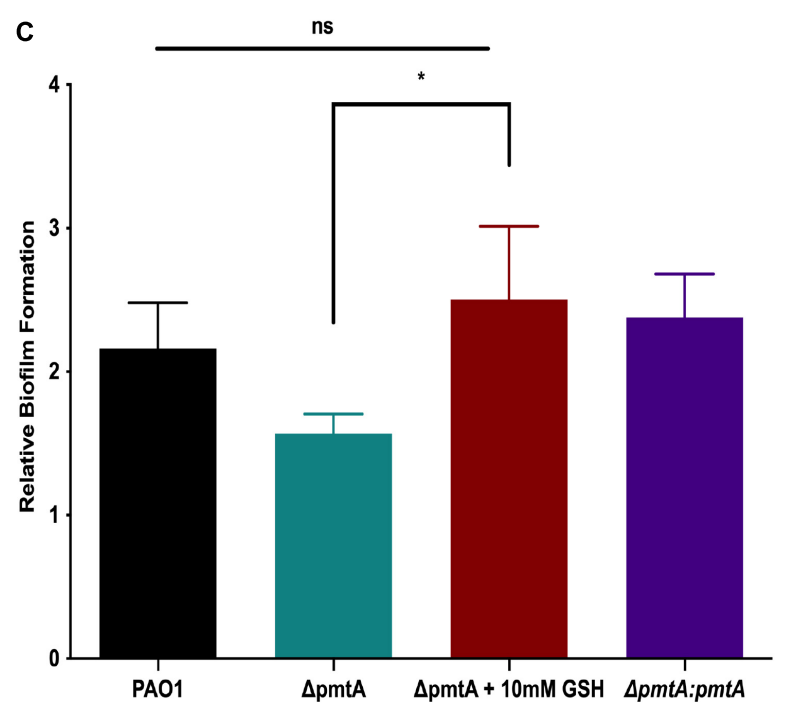

FIGURE 5 | PmtA is required for biofilm formation and the addition of antioxidant glutathione restores biofilm formation in $P$. aeruginosa $\triangle p m t A$ mutant. (A) Cultures of $P$. aeruginosa strains were grown overnight in LB medium and stained with crystal violet. Less crystal violet was observed in the $\triangle p m t A$ mutant when compared to wild-type and $\triangle p m t A$ :pmtA PAO1 strains. (B) Cultures of $P$. aeruginosa strains were grown overnight in LB media, diluted 1:20 in fresh LB medium and grown to $0.5 \mathrm{OD}_{600}$. After $24 \mathrm{~h}$ of growth, growth yield was measured at $\mathrm{OD}_{600}$. Biofilms were stained as previously described with minor modifications $\left(\mathrm{O}^{\prime} \mathrm{T}_{\mathrm{o}} \mathrm{le}, 2010\right)$ and crystal violet stain was measured at $\mathrm{OD}_{595}$. The OD reading after the addition of crystal violet was divided by the growth yield OD to calculate the relative biofilm formation. (C) GSH at $10 \mathrm{mM}$ was added to the LB media in the 96 well plate assay to determine if an antioxidant would rescue the biofilm formation defect in the $\triangle p m t A$ mutant. GSH restored the biofilm formation of the $\Delta p m t A$ mutant in the microtiter plate assay. An unpaired $t$-test analysis was performed $\left({ }^{*} p<0.05\right.$; $\left.{ }^{\star \star} p<0.01 ;{ }^{* \star \star} p<0.001 ;{ }^{\star \star \star \star} p<0.0001\right)$. The data are presented as the average of three biological replicates ( \pm standard error of the mean) and are representative of three separate experiments.

$\triangle p m t A: p m t A$ strains (Figure 7). Compared to the wild-type and $\triangle p m t A: p m t A$ PAO1 strains, the $\triangle p m t A$ mutant exhibited significantly lower MICs for cefepime and ciprofloxacin, indicating that PmtA promotes resistance to these antibiotics. However, no differences were observed among strains exposed to tetracycline, suggesting that PmtA does not affect $P$. aeruginosa growth in the presence of this antibiotic, to which it is naturally resistant.

\section{PmtA Expression Influences Pseudomonas aeruginosa Virulence in vivo}

The correlation between PmtA expression and the crucial virulence factors pyocyanin and biofilm formation indicates that
PmtA may contribute to the overall virulence of $P$. aeruginosa. To evaluate the virulence of the $\Delta p m t A$ mutant, we used $G$. mellonella larvae, a model system used to test the virulence of infectious bacteria and the innate immune response to cope with infection (Hoffmann, 1995; Mulcahy et al., 2011; Haller et al., 2014). Larvae were injected with $5 \mu \mathrm{L}$ of diluted cultures containing $10^{3}$ cells of the wild-type. $\triangle p m t A$, or $\triangle p m t A: p m t A$ strains. Survival of the larvae was evaluated 20, 24, 28 and $48 \mathrm{~h}$ after injection (Figure 8). At $20 \mathrm{~h}, 100 \%$ of the larvae injected with the $\Delta p m t A$ mutant survived compared to $10 \%$ of those injected with the wild-type strain, a $90 \%$ difference in survival rates. Compared to the $\triangle p m t A$ mutant, the larvae injected with the complemented strain showed a $42 \%$ decrease in survival at $20 \mathrm{~h}$. At $24 \mathrm{~h}$, the percent survival of larvae injected with the $\triangle p m t A$ mutant decreased to $52.5 \%$, while those injected 
A

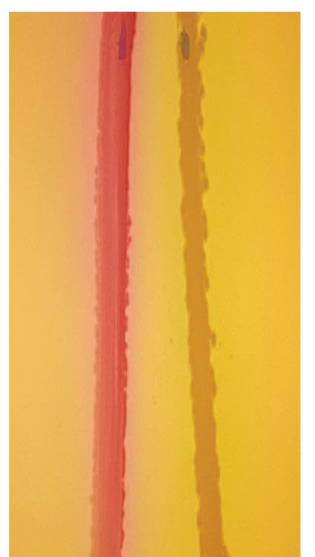

B

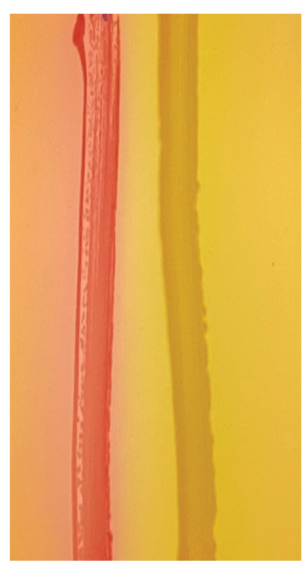

C

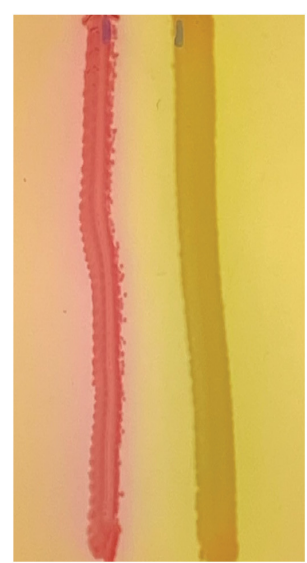

D

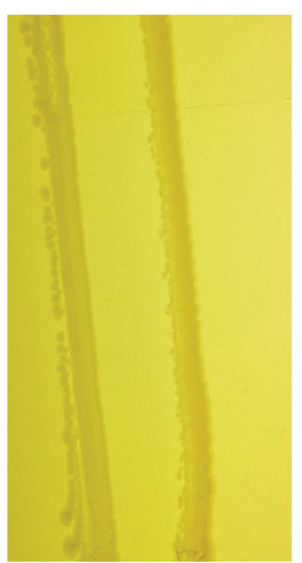

FIGURE 6 | PmtA does not affect acyl homoserine lactones levels. Wild-type (A), $\Delta p m t A$ (B), and $\Delta p m t A: p m t A$ (C). PAO1 (D) E. coli MC1061 strains were streaked out on MacConkey agar plates $0.75 \mathrm{~cm}$ apart from E. coli strain MG4. In the presence of AHL, lasR initiates the transcription of LasB-LacZ fusion protein resulting in beta-galactosidase in MG4, this causes colonies to change color from white to pink. All three strains caused the color change to pink suggesting that QS mediated by $\mathrm{AHL}$ is not inhibited by the absence of PmtA.
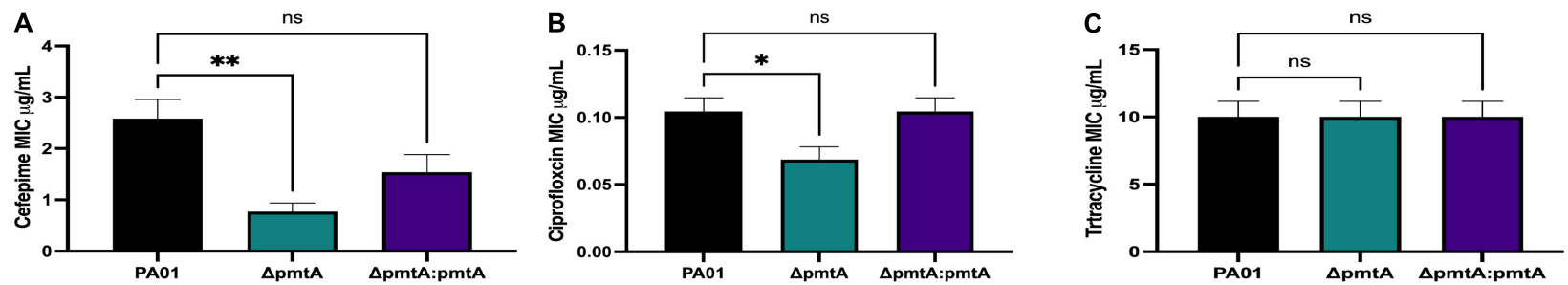

FIGURE 7 | The effects of PmtA on the Minimum Inhibitory Concentration (MIC) of cefepime, ciprofloxacin and tetracycline. Single colonies from the wild-type, $\Delta p m t A$, and $\Delta p m t A: p m t A$ PAO1 strains were each inoculated into $1 \mathrm{~mL}$ of LB. A sterile cotton swab was used to spread the culture over a LB plate. Liofilchem ${ }^{\circledR}$ MTS E-tests were placed separately onto the plates after inoculation for cefepime (A), ciprofloxacin (B) and tetracycline (C). When compared to wild-type and $\Delta p m t A: p m t A$ PAO1 strains, the $\triangle p m t A$ mutant was significantly more susceptible to cefepime and ciprofloxacin. One-way ANOVA analysis was performed $\left({ }^{\star} p<0.05 ;{ }^{* \star} p<0.01 ;{ }^{* \star *} p<0.001 ;{ }^{* \star \star *} p<0.0001\right.$. The data are presented as the average of three biological replicates $( \pm$ standard error of the mean) and are representative of three separate experiments.

with $\triangle p m t A: p m t A$ and wild-type was less than $20 \%$. These data indicate a role for PmtA in the virulence of $P$. aeruginosa.

\section{DISCUSSION}

Pyocyanin and biofilm formation are powerful virulence factors for $P$. aeruginosa, especially in invasive lung infections. Pyocyanin is easily diffusible through host membranes and can induce a variety of harmful cellular effects including neutrophil apoptosis, alter the expression of a number of cytokines and change the redox environment leading to the persistence of harmful oxidants produced by host polymorphonuclear leukocytes (PMNs) and macrophages (Usher et al., 2002; Managò et al., 2015; Hall et al., 2016). Here, we show for the first time that a bacterial MT, PmtA, can alter the amount of pyocyanin produced by $P$. aeruginosa, thereby influencing biofilm formation.

Pyocyanin can influence biofilm formation in two different ways. First, it can directly accept electrons from $\mathrm{NADH}$ or NADPH during aerobic respiration. This process passes electrons to the final electron donor to generate reactive oxygen species (e.g., superoxide and hydrogen peroxide) (Gonçalves and Vasconcelos, 2021). The oxidative stress caused by ROS production has been shown to cause a portion of the $P$. aeruginosa cell population to undergo autolysis, releasing eDNA, suggesting that pyocyanin can mediate eDNA production and help promote biofilm formation (Okshevsky and Meyer, 2015; Das et al., 2016; Malhotra et al., 2019). Pyocyanin has also been shown to influence biofilm formation by directly interacting with eDNA. This binding can mediate aggregation and cell-cell interaction within biofilms, stabilizing the biofilm structure (Das et al., 2013). eDNA is an essential component of the extracellular polymeric substance produced by $P$. aeruginosa and has been implicated in early attachment/biofilm formation and resistance to antimicrobials (Malhotra et al., 2019). The $\Delta p m t A$ mutant exhibited a defect in biofilm formation and/or stabilization, which is likely due to less pyocyanin availability. Interestingly, GSH restored both the pyocyanin levels and the biofilm defect in the $\Delta p m t A$ strain. GSH neutralizes oxidative stress, mediated by pyocyanin, and $P$. aeruginosa gsh mutants produce less pyocyanin (Van Laar et al., 2018). Together with previous data indicating that PmtA may be upregulated after oxidant exposure, these 


\section{$10^{\wedge} 3$}

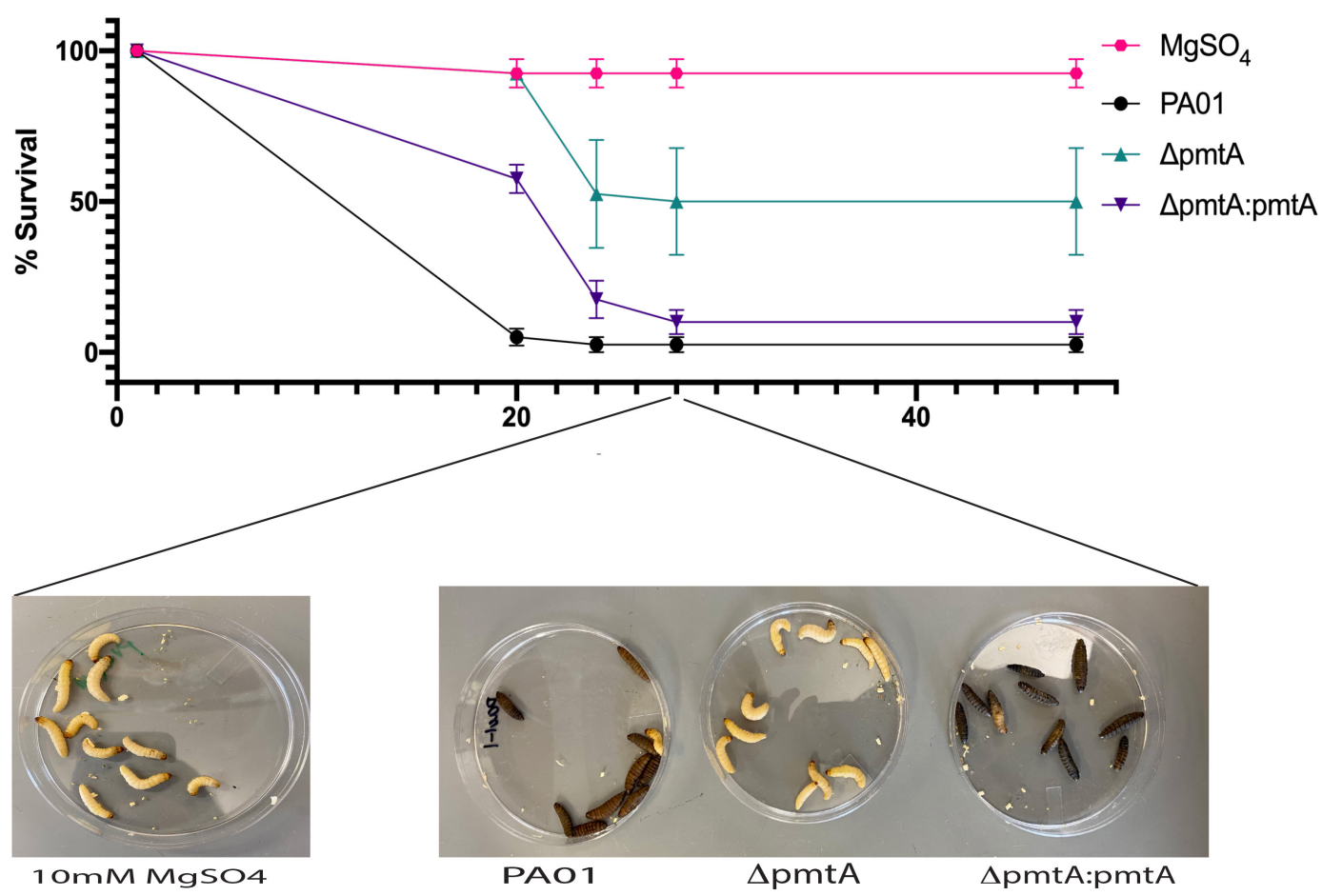

FIGURE 8 | Wild-type PAO1 is more lethal than the $\Delta p m t A$ mutant in a G. mellonella model. G. mellonella were injected with $5 \mu \mathrm{L}$ of $10^{3} / \mathrm{mL}$ of either $P$. aeruginosa wild-type, $\Delta p m t A$ and $\Delta p m t A: p m t A$ PAO1 strains or $10 \mathrm{mM}$ of $\mathrm{MgSO}_{4}$ (control). G. mellonella survival was observed for $48 \mathrm{~h}$ for all four groups, and percent survival was calculated. $\Delta p m t A$ had a higher survival percentage than PAO1 and $\Delta p m t A: p m t A$. The data are presented as the mean and standard deviation of the mean of three independent experiments ( $n=10$ /group).

results suggest a role for PmtA in relieving oxidative stress. Alternatively, PmtA may interact with $P$. aeruginosa-derived GSH to promote pyocyanin levels. Future studies will focus on investigating potential PmtA interactions.

We also observed a decrease in the expression of phzM, a gene that encodes for methyl-transferase. This enzyme helps convert phenazine-1-carboxylic acid to 5-methylphenazine-1carboxylic acid betaine allowing for the synthesis of pyocyanin. The low transcript levels of $p h z M$ detected in the $\Delta p m t A$ mutant compared to the wild-type strain correspond with the pyocyanin production phenotypes observed for these strains. Interestingly, PhzM expression is QS mediated, and QS has been shown to enhance the stress response in $P$. aeruginosa, allowing for less social cheating to occur in the population (García-Contreras et al., 2014; Chen et al., 2019). Although we did not observe a defect in AHL production within the $\Delta p m t A$ mutant, this does not rule out that PmtA could interact with one of the four QS systems. The mechanism by which PmtA influences phzM expression in early stationary cultures remains to be understood.

Low molecular-weight thiols are essential to the detoxification of oxidative stressors, and our data suggests that PmtA may play a role in relieving oxidative stress. Interestingly, the $\Delta p m t A$ mutant showed no growth defect when cultured in the presence of inhibitory levels of oxidative or heavy metal stressors, indicating that PmtA is not essential under these conditions. A potential reason for this lack of sensitivity may be the upregulation of yet to be identified proteins and enzymes involved in protection against these stresses that could compensate for the reduction in PmtA expression. In mycobacterial thiol mutants, defective for mycothiol and ergothioneine production, organic hydroperoxide resistance protein (Ohr) levels are upregulated to promote oxidative stress protection (Ta et al., 2011; Emani et al., 2013; Singh et al., 2016). Like mycobacteria, the absence of PmtA in the $\Delta p m t A$ strain may lead to upregulation of essential proteins for protection against oxidative stress. However, if this were the case, the phenotypic restoration that occurred upon the addition of GSH may not have been observed.

Alternatively, as $P$. aeruginosa has many systems dedicated to the regulation of oxidative and heavy metal stress (i.e., a catalase, siderophores, efflux pumps and metal reduction), these systems may compensate for the lack of PmtA in the $\Delta p m t A$ mutant, resulting in no defect being observed under the assayed growth conditions. Previous studies have shown similar results where the absence of GSH had no impact on sensitivity to hydrogen peroxide or the organic peroxide cumene hydroperoxide (CHP) (Van Laar et al., 2018). Some cyanobacteria also regulate MT production by SmtB, a zinc-dependent repressor. The PmtA operon does not encode metal-responsive regulators (Morby et al., 1993; Blindauer, 2008). Instead, the predicted operon includes two genes, one with unknown function and putatively 
short-chain dehydrogenase (Mao et al., 2009). In addition, Habjanič et al. (2020) noted that PflQ2 MT expression was elevated during stationary phase with or without the addition of metals in $P$. fluorescens Q2-87. The addition of metals at exponential phase did not induce transcription of MT, and increasing metal concentrations resulted in a decline in growth. At both exponential and stationary phases, we also did not observe any difference in growth among the assayed strains, supporting the conclusion that PmtA is not regulated by heavy metals.

Antibiotic resistance is a worldwide health crisis and the WHO has recently added carbapenem-resistant $P$. aeruginosa to the list of pathogens for which there is a critical need for new antibiotic treatments (Pang et al., 2019). Prolonged persistence of oxidants at low levels may be advantageous for antibiotic protection in bacteria. $P$. aeruginosa expresses efflux pumps that serve to expel antibiotics from the bacterial cell and thus protect the cell. An oxidant-sensitive negative regulator, MexR, controls the expression of this efflux pump (Poole et al., 1996). Oxidants cause a conformational change in MexR, which drives efflux pump transcription (Chen et al., 2008). Therefore, a decrease in extracellular oxidant production could limit the effective expression of the efflux pump and make $P$. aeruginosa more susceptible to antibiotics. In the present study, we showed that the $\Delta p m t A$ mutant is more susceptible to two of the conventional antibiotics used for treatments of $P$. aeruginosa in cystic fibrosis patients. These results suggest that PmtA expression could make $P$. aeruginosa more resistant to antibiotics.

Interestingly, the effects of tetracycline were unchanged in all three strains. The difference in sensitivity could be due to the difference in antibiotic resistance classifications (i.e., intrinsic, acquired, and adaptive). Resistance to tetracycline would fall under an intrinsic classification and would include outer membrane permeability, expression efflux pumps or production of antibiotic-inactivating enzyme, whereas the development of resistance to cefepime and ciprofloxacin would fall under adaptive resistance (Reygaert, 2018). One hypothesis is that this tolerance is linked to biofilm protection, which limits the access of the antibiotic. Since the $\Delta p m t A$ mutant has a weakened ability to form biofilms, this phenomenon could explain the increased sensitivity to these two therapeutics. Alternatively, pyocyanin levels have been linked to MDR $P$. aeruginosa strains, with higher levels being found in MDR vs. non-MDR strains (Gajdács et al., 2021). This suggests that the $\Delta p m t A$ mutant's lower levels of pyocyanin could convey sensitivity, possibly due to a decrease in oxidant production.

The substantial decrease in pyocyanin levels and biofilm formation in the PmtA-deficient $\triangle p m t A$ strains suggests that this strain is less able to influence the host immune response and thus would be less able to establish a persistent infection. The results reported in the present study suggest that a deficiency in PmtA expression results in a significant change to $P$. aeruginosa virulence. We used $G$. mellonella larvae to assess the importance of PmtA in the innate immune response as a virulence factor. Our results showed the $\Delta p m t A$ mutant was $90 \%$ less virulent than wild-type PAO1. The virulence was restored in the complemented strain $\triangle p m t A: p m t A$, indicating that PmtA is an important protein for establishing infection in a host. Taken together, these results indicate that PmtA can affect $P$. aeruginosa virulence by influencing pyocyanin expression.

It appears likely that therapeutic manipulation of PmtA during infection could decrease pyocyanin production and reduce biofilm formation. This disruption of PmtA may be an easier therapeutic target than interfering with the several genes involved in pyocyanin expression production. This work also demonstrates the complex regulation of pyocyanin, which is governed in part by PmtA expression. Since pyocyanin expression and biofilm formation could be rescued with an exogenous antioxidant addition, PmtA likely controls the transcription of the genes involved in pyocyanin expression by altering the redox environment. A change in the redox environment could also affect the expression of other proteins involved in $P$. aeruginosa virulence. The results of the present study show that PmtA affects the expression of virulence factors involved in establishing $P$. aeruginosa infection. Thus, targeting PmtA may represent a new, innovative option in treating the human pathogen $P$. aeruginosa.

\section{DATA AVAILABILITY STATEMENT}

The original contributions presented in the study are included in the article/Supplementary Material, further inquiries can be directed to the corresponding authors.

\section{AUTHOR CONTRIBUTIONS}

AT planned, performed the deletion and complementation of $p m t A$, bacterial growth curves, pyocyanin extraction, qPCR, biofilm assay, waxworm infection, and wrote the manuscript. KP planned, performed bacterial growth curves, pyocyanin extraction, biofilm assay, waxworm infections, and wrote the manuscript. ML planned, supervised the experiments, and helped wrote the manuscript. MM-M planned, performed acyl homoserine lactone-dependent QS assay, bacterial growth curves, MIC determination, and waxworm infections, and helped wrote the manuscript. CM performed bacterial growth curves, pyocyanin extractions, and GSH recovery experiments. JM planned deletion, complementation of mutants, and edit manuscript. JG planned and discussed the experiments. All authors contributed to the article and approved the submitted version.

\section{ACKNOWLEDGMENTS}

We want to thank Dr. Schweizer for providing the pEX18Gm plasmid.

\section{SUPPLEMENTARY MATERIAL}

The Supplementary Material for this article can be found online at: https://www.frontiersin.org/articles/10.3389/fmicb. 2021.789765/full\#supplementary-material 
Supplementary Figure $\mathbf{S 1}$ | Schematic representation of $P$. aeruginosa pmtA knock-out strain construction. Schematic indicates the generation of the $\Delta p m t A$ mutant.

Supplementary Figure S2 | Confirmation of Tn7 insertion into clean deletion mutant by colony PCR. Lanes 2, 3, and 4 used the pTn7GM_F and pTnGM_R primers, while lanes 5 and 6 used the PPA2140_Tn7_F and PA2140_Tn7_R primers with GoTaq Green Master Mix as previously described. Lane 1, 100 bp ladder; lane 2, pUC18R6K-mini-Tn7T-Gm-promoterPA2140 transformant (979 bp); lane 3, complemented $\Delta p m t A$ mutant ( $\Delta p m t A$ :pmtA); lane 4 , pUC18R6K-mini-Tn7T-Gm empty vector (261 bp); lane 5, wild-type PAO1 (781 bp); and lane 6, complemented $\Delta p m t A$ mutant $\Delta p m t A: p m t A$ (781 bp).

Supplementary Figure S3 $\mid P$. aeruginosa strains PAO1, $\Delta p m t A$, and $\Delta p m t A$ :pmtA grow at similar rates. Single colonies of the $P$. aeruginosa strains were each grown overnight in $5 \mathrm{~mL}$ of $\mathrm{LB}$ media at $37^{\circ} \mathrm{C}$ with vigorous shaking. These cultures were transferred to (A). $50 \mathrm{~mL}$ LB media at a dilution of 1:100 and grown at $37^{\circ} \mathrm{C}$ with vigorous shaking. Growth was measured at 2, 4, 6, 24, and $30 \mathrm{~h}$ by removing $250 \mu \mathrm{L}$ of culture in triplicate and transferring to a NUNC 96 well plate. The plate was measured using a Spectramax microplate reader. (B) $1 \mathrm{~mL}$ of 1:100 diluted cultures were transferred to a NUNC 96 well plate in triplicate. Growth was monitored over $15 \mathrm{~h}$ at $37^{\circ} \mathrm{C}$ with $5 \mathrm{~s}$ shaking every $5 \mathrm{~min}$ in a Spectramax microplate reader at $\mathrm{OD}_{600}$. (C) Cultures were grown in $\mathrm{M} 9$ salts media in a 24 well plate and monitored over $15 \mathrm{~h}$ at $37^{\circ} \mathrm{C}$ with $5 \mathrm{~s}$ shaking every $1 \mathrm{~h}$ in a Spectramax microplate reader at $\mathrm{OD}_{600}$. The data are presented as the average of three biological replicates ( \pm standard error of the mean) and are representative of three separate experiments.

Supplementary Figure S4 | PmtA is not required for survival in oxidative stress conditions. Overnight cultures were diluted at 1:100 and grown for $2 \mathrm{~h}$. Cultures were adjusted to $0.08 \mathrm{OD}$ at $\mathrm{OD}_{600} .600 \mu \mathrm{L}$ of culture in triplicate were put in a 24-well plate containing either LB (A) or M9 salt media (B). Hydrogen peroxide to

\section{REFERENCES}

Aedekerk, S., Diggle, S. P., Song, Z., Høiby, N., Cornelis, P., Williams, P., et al. (2005). The MexGHI-OpmD multidrug efflux pump controls growth, antibiotic susceptibility and virulence in Pseudomonas aeruginosa via 4-quinolonedependent cell-to-cell communication. Microbiology 151, 1113-1125. doi: 10 1099/mic.0.27631-0

Behzadi, P., Baráth, Z., and Gajdács, M. (2021). It's not easy being green: a narrative review on the microbiology, virulence and therapeutic prospects of multidrug-resistant Pseudomonas aeruginosa. Antibiotics 10:42. doi: 10.3390/ ANTIBIOTICS10010042

Blindauer, C. A. (2008). Metallothioneins with unusual residues: histidines as modulators of zinc affinity and reactivity. J. Inorgan. Biochem. 102, 507-521. doi: 10.1016/j.jinorgbio.2007.10.032

Blindauer, C. A. (2011). Bacterial metallothioneins: past, present, and questions for the future. J. Biol. Inorgan. Chem. 16, 1011-1024. doi: 10.1007/s00775-0110790-y

Blindauer, C. A. (2015). Advances in the molecular understanding of biological zinc transport. Chem. Commun. 51, 4544-4563. doi: 10.1039/c4cc10174j

Borghesi, L. A., and Lynes, M. A. (1996). Nonprotective effects of extracellular metallothionein. Toxicol. Appl. Pharmacol. 139, 6-14. doi: 10.1006/taap.1996. 0137

Chen, H., Hu, J., Chen, P. R., Lan, L., Li, Z., Hicks, L. M., et al. (2008). The Pseudomonas aeruginosa multidrug efflux regulator MexR uses an oxidationsensing mechanism. Proc. Natl. Acad. Sci. U.S.A. 105, 13586-13591. doi: 10. 1073/pnas.0803391105

Chen, R., Déziel, E., Groleau, M.-C., Schaefer, A. L., and Greenberg, E. P. (2019). Social cheating in a Pseudomonas aeruginosa quorum-sensing variant. Proc. Natl. Acad. Sci. U.S.A. 116, 7021-7026. doi: 10.1073/PNAS.1819801116

Choi, K. H., and Schweizer, H. P. (2006). Mini-Tn7 insertion in bacteria with single AttTn7 sites: example Pseudomonas aeruginosa. Nat. Protoc. 1, 153-161. doi: $10.1038 /$ nprot.2006.24

Chuanchuen, R., Narasaki, C. T., and Schweizer, H. P. (2002). The MexJK efflux pump of Pseudomonas aeruginosa requires OprM for antibiotic efflux but not a final concentration of $10 \mathrm{mM}$ or water was added to the wells. (A) Growth was measured at $\mathrm{OD}_{600}$ every hour with shaking before and after measurement in a Spectramax microplate reader. The data are presented as the average of three biological replicates ( \pm standard error of the mean) and are representative of three separate experiments.

Supplementary Figure S5 | PmtA is not required for survival in heavy metal stress conditions. Overnight cultures were diluted at 1:100 and grown for $2 \mathrm{~h}$ in LB or M9 salt media. Cultures were adjusted to $0.08 \mathrm{OD}$ at $\mathrm{OD}_{600} .600 \mu \mathrm{L}$ of culture in triplicate were put in a 24-well plate. Zinc chloride $\mathbf{( A , B )}$ to a final concentration of $200 \mu \mathrm{M}$, or cadmium chloride (C,D) to a final concentration of $100 \mu \mathrm{M}$. Growth was measured at $\mathrm{OD}_{600}$ every hour with shaking before and after measurement in a Spectramax microplate reader. The data are presented as the average of three biological replicates ( \pm standard error of the mean) and are representative of three separate experiments.

Supplementary Figure S6 | PmtA does not play a role in iron uptake. Wild-type PAO1, $\Delta p m t A$, and $\Delta p m t A: p m t A$ cultures were grown overnight in $\mathrm{M} 9$ salt media, diluted at 1:100 and grown in M9 salt media until they reached an $\mathrm{OD}_{600}$ of 0.08 . $600 \mu \mathrm{L}$ of culture in triplicate were put in a 24-well plate. M9 salt media (orange), EDDA to a final concentration of $150 \mu \mathrm{M}$ (blue), EDDA $+\mathrm{FeCl}_{2}$ (black) to a final concentration of $150 \mu \mathrm{M}$ and $50 \mu \mathrm{g}$, or EDDA + Transferrin (purple) to a final concentration of $150 \mu \mathrm{M}$ and $200 \mu \mathrm{g}$. Growth was measured at $\mathrm{OD}_{600}$ every hour with shaking before and after measurement in a Spectramax microplate reader. The circles indicate PAO1, the squares indicate $\triangle p m t A$, and the triangles indicate $\Delta p m t A: p m t A$. The data are presented as the average of three biological replicates ( \pm standard error of the mean) and are representative of three separate experiments.

Supplementary Table S1 | Bacterial strains and plasmids used in the present study.

Supplementary Table S2 | Primers used in the present study.

for efflux of triclosan. J. Bacteriol. 184, 5036-5044. doi: 10.1128/JB.184.18.50365044.2002

Das, T., Ibugo, A. I., Klare, W., and Manefield, M. (2016). "Role of pyocyanin and extracellular DNA in facilitating Pseudomonas aeruginosa biofilm formation," in Microbial Biofilms - Importance and Applications, eds D. Dhanasekaran and N. Thajuddin (London: InTech), 23-42.

Das, T., Kutty, S. K., Kumar, N., and Manefield, M. (2013). Pyocyanin facilitates extracellular DNA binding to Pseudomonas aeruginosa influencing cell surface properties and aggregation. PLoS One 8:e0058299. doi: 10.1371/journal.pone. 0058299

Devisscher, L., Hindryckx, P., Lynes, M. A., Waeytens, A., Cuvelier, C., De Vos, F., et al. (2014). Role of metallothioneins as danger signals in the pathogenesis of colitis. J. Pathol. 233, 89-100. doi: 10.1002/PATH.4330

Emani, C. S., Williams, M. J., Wiid, I. J., Hiten, N. F., Viljoen, A. J., Pietersen, R. D. D., et al. (2013). Ergothioneine is a secreted antioxidant in mycobacterium smegmatis. Antimicrob. Agents Chemother. 57, 3202-3207. doi: 10.1128/AAC. 02572- 12

Essar, D. W., Eberly, L., Hadero, A., and Crawford, I. P. (1990). Identification and characterization of genes for a second anthranilate synthase in Pseudomonas aeruginosa: interchangeability of the two anthranilate synthases and evolutionary implications. J. Bacteriol. 172, 884-900. doi: 10.1128/JB.172.2. 884-900.1990

Frank, L. H., and Demoss, R. D. (1959). On the biosynthesis of pyocyanine. J. Bacteriol. 16, 576-583. doi: 10.1128/jb.77.6.776-782. 1959

Gajdács, M., Baráth, Z., Kárpáti, K., Szabó, D., Usai, D., Zanetti, S., et al. (2021). No correlation between biofilm formation, virulence factors, and antibiotic resistance in Pseudomonas aeruginosa: results from a laboratorybased in vitro study. Antibiotics 10:1134. doi: 10.3390/ANTIBIOTICS100 91134

García-Contreras, R., Nuñ Ez-Ló Pez, L., Jasso-Chávez, R., Kwan, B. W., Belmont, J. A., Rangel-Vega, A., et al. (2014). Quorum sensing enhancement of the stress response promotes resistance to quorum quenching and prevents social cheating. ISME J. 9, 115-125. doi: 10.1038/ismej.2014.98 
Gellatly, S. L., and Hancock, R. E. W. (2013). Pseudomonas aeruginosa: new insights into pathogenesis and host defenses. Pathog. Dis. 67, 159-173. doi: 10.1111/ 2049-632X.12033

Goldová, J., Ulrych, A., Hercík, K., and Branny, P. (2011). A eukaryotic-type signalling system of Pseudomonas aeruginosa contributes to oxidative stress resistance, intracellular survival and virulence. BMC Genomics 12:437. doi: 10.1186/1471-2164-12-437

Gonçalves, T., and Vasconcelos, U. (2021). Colour me blue: the history and the biotechnological potential of pyocyanin. Molecules 26:927. doi: 10.3390/ molecules 26040927

Habjanič, J., Mathew, A., Eberl, L., and Freisinger, E. (2020). Deciphering the enigmatic function of Pseudomonas metallothioneins. Front. Microbiol. 11:1709. doi: 10.3389/fmicb.2020.01709

Hall, S., McDermott, C., Anoopkumar-Dukie, S., McFarland, A. J., Forbes, A., Perkins, A. V., et al. (2016). Cellular effects of pyocyanin, a secreted virulence factor of Pseudomonas aeruginosa. Toxins 8:236. doi: 10.3390/TOXINS8080236

Haller, S., Limmer, S., and Ferrandon, D. (2014). Assessing Pseudomonas Virulence with a Nonmammalian Host: Drosophila Melanogaster. New York, NY: Humana Press, 723-740.

Hassan, H. M., and Fridovich, I. (1980). Mechanism of the antibiotic action of pyocyanine. J. Bacteriol. 141, 156-163. doi: 10.1128/jb.141.1.156-163.1980

Hentzer, M., Eberl, L., and Givskov, M. (2005). Transcriptome analysis of Pseudomonas aeruginosa biofilm development: anaerobic respiration and iron limitation. Biofilms 2, 37-61. doi: 10.1017/S1479050505001699

Hmelo, L. R., Borlee, B. R., Almblad, H., Love, M. E., Randall, T. E., Tseng, B. S., et al. (2015). Precision-engineering the Pseudomonas aeruginosa genome with two-step allelic exchange. Nat. Protoc. 10, 1820-1841. doi: 10.1038/nprot.2015. 115

Hoang, T. T., Karkhoff-Schweizer, R. R., Kutchma, A. J., and Schweizer, H. P. (1998). A broad-host-range F1p-FRT recombination system for site-specific excision of chromosomally-located DNA sequences: application for isolation of unmarked Pseudomonas aeruginosa mutants. Gene 212, 77-86. doi: 10.1016/ S0378-1119(98)00130-9

Hoffmann, J. A. (1995). Innate Immunity of Insects. Curr. Opin. Immunol. 7, 4-10. doi: 10.1016/0952-7915(95)80022-0

Jacobs, M. A., Alwood, A., Thaipisuttikul, I., Spencer, D., Haugen, E., Ernst, S., et al. (2003). Comprehensive transposon mutant library of Pseudomonas aeruginosa. Proc. Natl. Acad. Sci. U.S.A. 100:14339. doi: 10.1073/PNAS.20362 82100

Lau, G. W., Hassett, D. J., Ran, H., and Kong, F. (2004a). The role of pyocyanin in Pseudomonas aeruginosa infection. Trends Mol. Med. 10, 599-606.

Lau, G. W., Ran, H., Kong, F., Hassett, D. J., and Mavrodi, D. (2004b). Pseudomonas aeruginosa pyocyanin is critical for lung infection in mice. Infect. Immun. 72, 4275-4278. doi: 10.1128/IAI.72.7.4275-4278.2004

Laukens, D., Waeytens, A., De Bleser, P., Cuvelier, C., and De Vos, M. (2009). Human metallothionein expression under normal and pathological conditions: mechanisms of gene regulation based on in silico promoter analysis. Crit. Rev. Eukaryotic Gene Express. 19, 301-317. doi: 10.1615/ CRITREVEUKARGENEEXPR.V19.I4.40

Lynes, M. A., Borghesi, L. A., Youn, J., and Olson, E. A. (1993). Immunomodulatory activities of extracellular metallothionein. I. metallothionein effects on antibody production. Toxicology 85, 161-177. doi: 10.1016/0300-483X(93)90040-Y

Magalhães, A. P., Lopes, S. P., and Pereira, M. O. (2016). Insights into cystic fibrosis polymicrobial consortia: the role of species interactions in biofilm development, phenotype, and response to in-use antibiotics. Front. Microbiol. 7:2146. doi: 10.3389/FMICB.2016.02146

Malhotra, S., Hayes, D. Jr., and Wozniak, D. J. (2019). Cystic fibrosis and Pseudomonas aeruginosa: the host-microbe interface. Clin. Microbiol. Rev. 32:e00138-18. doi: 10.1128/CMR.00138-18

Managò, A., Becker, K. A., Carpinteiro, A., Wilker, B., Soddemann, M., Seitz, A. P., et al. (2015). Pseudomonas aeruginosa pyocyanin induces neutrophil death via mitochondrial reactive oxygen species and mitochondrial acid sphingomyelinase. Antioxid. Redox Signal. 22, 1097-1110. doi: 10.1089/ARS. 2014.5979

Mao, F., Dam, P., Chou, J., Olman, V., and Xu, Y. (2009). DOOR: a database for prokaryotic operons. Nucleic Acids Res. 37, D459-D463. doi: 10.1093/nar/ gkn757
Maret, W., and Moulis, J.-M. (2013). The bioinorganic chemistry of cadmium in the context of its toxicity. Met. Ions Life Sci. 11, 1-29.

Marvig, R. L., Sommer, L. M., Molin, S., and Johansen, H. K. (2014). Convergent evolution and adaptation of Pseudomonas aeruginosa within patients with cystic fibrosis. Nat. Genet. 47, 57-64. doi: 10.1038/ng.3148

Mavrodi, D. V., Bonsall, R. F., Delaney, S. M., Soule, M. J., Phillips, G., and Thomashow, L. S. (2001). Functional analysis of genes for biosynthesis of pyocyanin and phenazine-1-carboxamide from Pseudomonas aeruginosa PAO1. J. Bacteriol. 183, 6454-6465. doi: 10.1128/JB.183.21.6454-6465. 2001

Meister, A., and Anderson, M. E. (1983). Glutathione. Annu. Rev. Biochem. 52, 711-760. doi: 10.1146/annurev.bi.52.070183.003431

Moradali, M. F., Ghods, S., and Rehm, B. H. A. (2017). Pseudomonas aeruginosa lifestyle: a paradigm for adaptation, survival, and persistence. Front. Cell. Infect. Microbiol. 7:39. doi: 10.3389/FCIMB.2017.00039

Morby, A. P., Turner, J. S., Huckle, J. W., and Robinson, N. J. (1993). SmtB is a metal-dependent repressor of the cyanobacterial metallothionein gene SmtA: identification of a $\mathrm{Zn}$ inhibited DNA-protein complex. Nucleic Acids Res. 21, 921-925.

Mulcahy, H., Sibley, C. D., Surette, M. G., and Lewenza, S. (2011). Drosophila melanogaster as an animal model for the Study of Pseudomonas aeruginosa biofilm infections in vivo. PLoS Pathog. 7:e1002299. doi: 10.1371/journal.ppat. 1002299

Okshevsky, M., and Meyer, R. L. (2015). The role of extracellular DNA in the establishment, maintenance and perpetuation of bacterial biofilms. Crit. Rev. Microbiol. 41, 341-352.

O’Toole, G. A. (2010). Microtiter dish biofilm formation assay. J. Vis. Exp. 30:2437. doi: $10.3791 / 2437$

Pachori, P., Gothalwal, R., and Gandhi, P. (2019). Emergence of antibiotic resistance Pseudomonas aeruginosa in intensive care unit; a critical review. Genes Dis. 6:109. doi: 10.1016/J.GENDIS.2019.04.001

Pang, Z., Raudonis, R., Glick, B. R., Lin, T. J., and Cheng, Z. (2019). Antibiotic resistance in Pseudomonas aeruginosa: mechanisms and alternative therapeutic strategies. Biotechnol. Adv. 37, 177-192. doi: 10.1016/J.BIOTECHADV.2018.11. 013

Pearson, J. P., Passadori, L., Iglewskit, B. H., and Greenberg, E. P. (1995). A second N-Acylhomoserine lactone signal produced by Pseudomonas aeruginosa. Proc. Natl. Acad. Sci. U.S.A. 92, 1490-1494.

Poole, K., Tetro, K., Zhao, Q., Heinrichs, D. E., and Bianco, N. (1996). Expression of the multidrug resistance operon MexA-MexB-OprM in Pseudomonas aeruginosa: MexR encodes a regulator of operon expression. Antimicrob. Agents Chemother. 40, 2021-2028.

Qu, J., Cai, Z., Liu, Y., Duan, X., Han, S., Liu, J., et al. (2021). Persistent bacterial coinfection of a COVID-19 patient caused by a genetically adapted Pseudomonas aeruginosa chronic colonizer. Front. Cell. Infect. Microbiol. 11:641920. doi: 10.3389/FCIMB.2021.641920

Rada, B., and Leto, T. L. (2013). Pyocyanin effects on respiratory epithelium: relevance in Pseudomonas aeruginosa airway infections. Trends Microbiol. 21, 73-81. doi: 10.1016/j.tim.2012.10.004

Reygaert, W. C. (2018). An overview of the antimicrobial resistance mechanisms of bacteria. AIMS Microbiol. 4, 482-501. doi: 10.3934/microbiol.2018.3.482

Ruttkay-Nedecky, B., Nejdl, L., Gumulec, J., Zitka, O., Masarik, M., Eckschlager, T., et al. (2013). The role of metallothionein in oxidative stress. Int. J. Mol. Sci. 14, 6044-6066. doi: 10.3390/ijms14036044

Seed, K. D., and Dennis, J. J. (2008). Development of Galleria mellonella as an alternative infection model for the Burkholderia cepacia complex. Infect. Immun. 76, 1267-1275. doi: 10.1128/IAI.01249-07

Shao, X., Xie, Y., Zhang, Y., Liu, J., Ding, Y., Wu, M., et al. (2020). Novel therapeutic strategies for treating Pseudomonas aeruginosa infection. Expert Opin. Drug Discov. 15, 1403-1423. doi: 10.1080/17460441.2020.1803274

Shrivastava, P. S., and Ramasamy, J. (2018). World Health Organization releases global priority list of antibiotic-resistant bacteria to guide research, discovery, and development of new antibiotics. J. Med. Soc. 32:76. doi: 10.4103/JMS.JMS_ 25_17

Singh, A. R., Strankman, A., Orkusyan, R., Purwantini, E., and Rawat, M. (2016). Lack of mycothiol and ergothioneine induces different protective mechanisms in Mycobacterium smegmatis. Biochem. Biophys. Rep. 8, 100-106. doi: 10.1016/ j.bbrep. 2016.08 .006 
Ta, P., Buchmeier, N., Newton, G. L., Rawat, M., and Fahey, R. C. (2011). Organic hydroperoxide resistance protein and ergothioneine compensate for loss of mycothiol in Mycobacterium smegmatis mutants. J. Bacteriol. 193, 1981-1990. doi: 10.1128/JB.01402-10

Tacconelli, E., Carrara, E., Savoldi, A., Kattula, D., and Burkert, F. (2013). Global Priority List Of Antibiotic-Resistant Bacteria To Guide Research, Discovery, And Development Of New Antibiotics. Geneva: WHO.

Tsai, C. J. Y., Loh, J. M. S., and Proft, T. (2016). Galleria mellonella infection models for the study of bacterial diseases and for antimicrobial drug testing. Virulence 7, 214-229.

Ulrich, K., and Jakob, U. (2019). The role of thiols in antioxidant systems. Free Radic. Biol. Med. 140, 14-27.

Usher, L. R., Lawson, R. A., Geary, I., Taylor, C. J., Bingle, C. D., Taylor, G. W., et al. (2002). Induction of neutrophil apoptosis by the Pseudomonas aeruginosa exotoxin pyocyanin: a potential mechanism of persistent infection. J. Immunol. 168, 1861-1868. doi: 10.4049/jimmunol.168.4.1861

Van Laar, T. A., Esani, S., Birges, T. J., Hazen, B., Thomas, J. M., and Rawat, M. (2018). Pseudomonas aeruginosa GshA mutant is defective in biofilm formation, swarming, and pyocyanin production. mSphere 3:e00155-18. doi: 10.1128/ mSphere.00155-18

Waite, R. D., Paccanaro, A., Papakonstantinopoulou, A., Hurst, J. M., Saqi, M., Littler, E., et al. (2006). Clustering of Pseudomonas aeruginosa transcriptomes from planktonic cultures, developing and mature biofilms reveals distinct expression profiles. BMC Genomics 7:162. doi: 10.1186/1471-2164-7-162

Wong, M., Wong, D., and Malhotra, S. (2021). Intravenous fosfomycin as salvage therapy for osteomyelitis caused by multidrug-resistant Pseudomonas aeruginosa. Am. J. Health-Syst. Pharm. [Epub ahead of print]. doi: 10.1093/ AJHP/ZXAB294
Yin, X., Knecht, D. A., and Lynes, M. A. (2005). Metallothionein mediates leukocyte chemotaxis. BMC Immunol. 6:21. doi: 10.1186/1471-2172-6-21

Youn, J., Borghesi, L. A., Olson, E. A., and Lynes, M. A. (1995). Immunomodulatory activities of extracellular metallothionein. II. Effects on macrophage functions. J. Toxicol. Environ. Health 45, 397-413. doi: 10.1080/15287399509532004

Zaborin, A., Gerdes, S., Holbrook, C., Liu, D. C., Zaborina, O. Y., and Alverdy, J. C. (2012). Pseudomonas aeruginosa overrides the virulence inducing effect of opioids when it senses an abundance of phosphate. PLoS One 7:e34883. doi: 10.1371/journal.pone.0034883

Conflict of Interest: The authors declare that the research was conducted in the absence of any commercial or financial relationships that could be construed as a potential conflict of interest.

Publisher's Note: All claims expressed in this article are solely those of the authors and do not necessarily represent those of their affiliated organizations, or those of the publisher, the editors and the reviewers. Any product that may be evaluated in this article, or claim that may be made by its manufacturer, is not guaranteed or endorsed by the publisher.

Copyright (c) 2021 Thees, Pietrosimone, Melchiorre, Marden, Graf, Lynes and MaltzMatyschsyk. This is an open-access article distributed under the terms of the Creative Commons Attribution License (CC BY). The use, distribution or reproduction in other forums is permitted, provided the original author(s) and the copyright owner(s) are credited and that the original publication in this journal is cited, in accordance with accepted academic practice. No use, distribution or reproduction is permitted which does not comply with these terms. 